Index of Surface-Water Records to December 31, 1963 Hawaii and Other Pacific Areas 



\title{
Index of Surface-Water Records to December 31, 1963 Hawaii and Other Pacific Areas
}

\author{
By H. P. Eisenhuth
}

父

Goological Survey Circular 515 
United States Department of the Interior STEWART L. UDALL, SECRETARY

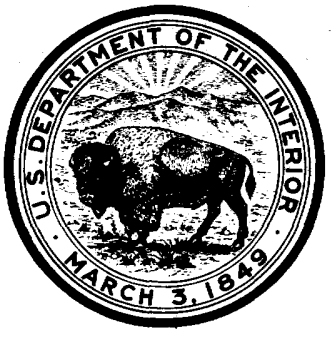

Geological Survey

THOMAS B. NOLAN, Director

X 


\title{
Index of Surface-Water Records to December 31, 1963
}

\author{
Hawaii and Other Pacific Areas
}

\author{
By H. P. Eisenhuth
}

INTRODUCTION

This report lists the streamflow stations in Hawail and other Pacific areas for which records have been or are to be published in reports of the Geological Survey for periods through December 31 , 1963. It supersedes Geological Survey Circular 395.

Basic data on surface-water supply of the United States prior to 1961 have been published in an annual series of water-supply papers consisting of several volumes, one of which was for Hawa11. Water-Supply Paper 1751, entitled "Surface Water Supply of Mariana, Caroline and Samoa Islands through June 1960" published data for other Pacific areas.

Beginning in 1961, the annual serles of water-supply papers on surface-water supply was changed to a 5-year series. Records for the period $1961-65$ will be published in a water-supply paper entitled "Surface Water Supply of Hawail and other Paclfic Areas." In order to meet interim requirements, beginning July 1 , 1960, the Hawali district issues reports annually containing streamflow records for Hawali and other Pacific areas.

In addition to the continuous-record gaging stations, this index includes crest-stage and lowflow partial-record stations. A continuous-record station is a gaging station on a stream or reservoir for which the discharge, stage, or contents is published on a daily, weekly, or monthly basis for a continuous period of time. A crest-stage partial-record station is a streamflow station for which only the annual maximum discharge is published over a period of years for use in floodflow analyses. A low-flow partial-record station is a streamflow station for which only discharge measurements made at base flow, when streamflow is primarily from ground-water storage, are published. Measurements are generally collected over a period of five years or more for use in low-flow analyses. Discharge measurements have been made at many sites and published as miscellaneous measurements. Such measurements are not included in this index except those made in earlier years at sites now classified as low-flow partial-record stations.

An alphabetical list of streams is given on pages 21-24.

\section{DOWNSTREAM ORDER}

The stations in this index are listed in the downstream order used in the series of watersupply papers on surface-water supply of Hawail since 1951. Starting at the headwater of each stream all stations are listed in a downstream direction. Tributary streams are indicated by indention and are inserted between main-stem stations in the order in which they enter the main stream. To indicate the rank of any tributary on which a record is available and the stream to which it is immediately tributary, each indention in the listing of stations represents one rank. A stream name, only, is inserted where necessary for the purpose of showing the proper rank or order of tributarles.

\section{STATION NAMES}

Station names are given in their most recently published form. Parentheses around part of a station name indicate that the enclosed word or words were used in an earlier published name of the station or as an alternate name. Parenthetical explanations are also used to indicate that a stream of a different name is the head of the main stream by use of "(head of name of the main stream has changed by use of "(continuation of RIver)" River)" and that the

\section{STATION NUMBER}

As an added means of identification, each continuous- and partial-record station has been assigned a station number. The numbers have been assigned in the same downstream order used in this index. In assigning station numbers, gaps were left to allow for new stations that may be established; hence the numbers are not consecutive. The first two digits of the complete number for each station identify the area, they are 40 for all stations in this report. The number given consists of only the essential digits of the complete number. For example, for a station with the complete number 40-0950.00, the station number shown in this index is 950 . 


\title{
DRAINAGE AREA
}

The drainage area, in square miles, is the latest figure published or otherwise avallable at this time.

\author{
PERIODS OF RECORD
}

Under "Periods of record" are three columns. The first column, "Daily or monthly figures," shows the periods of record for continuous-record gaging stations. The dates given are the calendar years in which records began or ended; breaks of less than a year are not shown. For example, if a record began in October 1923, ended in April 1932, began again in March 1933, and ended in September 1944, the period of record would be shown as 1923-44.

The second column, "Annual peaks," shows the period of record for crest-stage partialrecord stations. The dates given are fiscal years for which the annual maximum is avallable. The fiscal year begins July 1 and ends June 30. In listing the fiscal year, only one date is shown; for example, 1952 stands for the fiscal year July 1, 1951, to June 30, 1952 .

The third column "Low-flow measurements," shows the period of record for low-flow partialrecord stations, the dates given are the fiscal years in which base-flow measurements were made at low-flow partial-record stations; breaks of less than two years are not shown. Prior to the 1958 water year, such measurements were published in tables headed "Miscellaneous discharge measurements" or "Discharge measurements at sites other than gaging stations." Many discharge measurements have been made at miscellaneous sites which are not listed in this index because the data collected were not sufficient to qualify the site as a low-flow partial-record station.

A date followed by only a dash shows that the station was continued in operation beyond December 31, 1963. A date followed by a period indicates discontinuance. A date followed by a semicolon indicates a break in the collection of records. 


\begin{tabular}{|c|c|c|c|c|c|}
\hline \multirow[b]{2}{*}{ Station name } & \multirow{2}{*}{$\begin{array}{r}\text { Station } \\
\text { number }\end{array}$} & \multirow{2}{*}{$\begin{array}{c}\text { Drainage area } \\
(\text { sq mi })\end{array}$} & \multicolumn{3}{|c|}{ Periods of record } \\
\hline & & & $\begin{array}{l}\text { Dally or monthly } \\
\text { figures }\end{array}$ & Annual peaks & $\begin{array}{c}\text { Low-flow } \\
\text { measurements }\end{array}$ \\
\hline 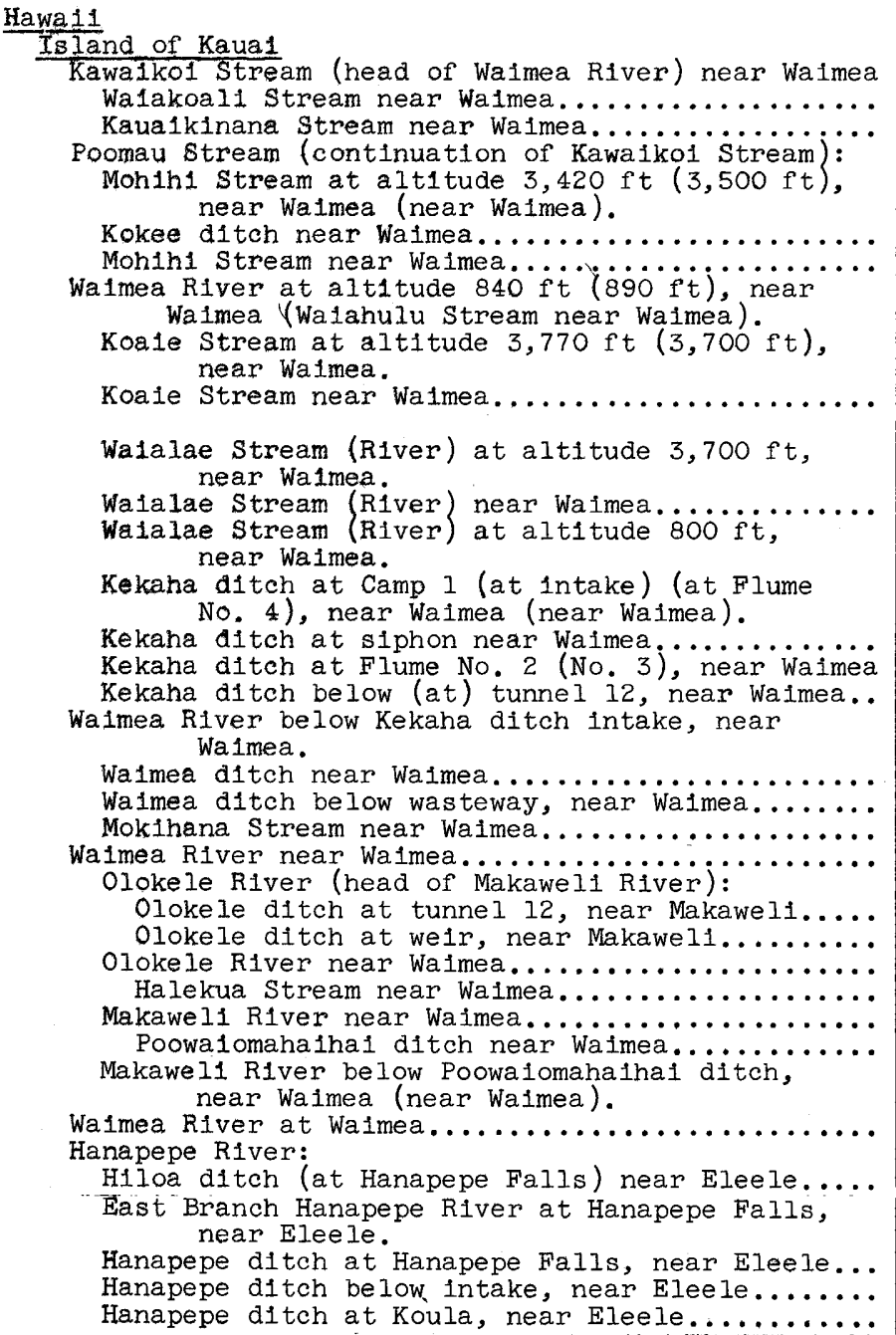 & $\begin{array}{l}130 \\
140 \\
150 \\
160 \\
170 \\
180 \\
\\
190 \\
\\
200 \\
210 \\
\\
220 \\
\\
240 \\
250 \\
270 \\
280 \\
\\
290 \\
291 \\
295 \\
310 \\
\\
320 \\
330 \\
340 \\
350 \\
360 \\
370 \\
371\end{array}$ & $\begin{array}{c}24.1 \\
a 1.7 \\
1.17 \\
\text { a1.6 } \\
- \\
\text { a2.2 } \\
20.0 \\
3.33 \\
11.7 \\
\text { a2.5 } \\
\text { a3.5 } \\
8.75 \\
- \\
- \\
- \\
45.0 \\
- \\
4.77 \\
57.8 \\
- \\
\text { a } \\
\text { a.9 } \\
\text { a25 } \\
\text { a25 } \\
88.0 \\
- \\
- \\
- \\
-\end{array}$ & 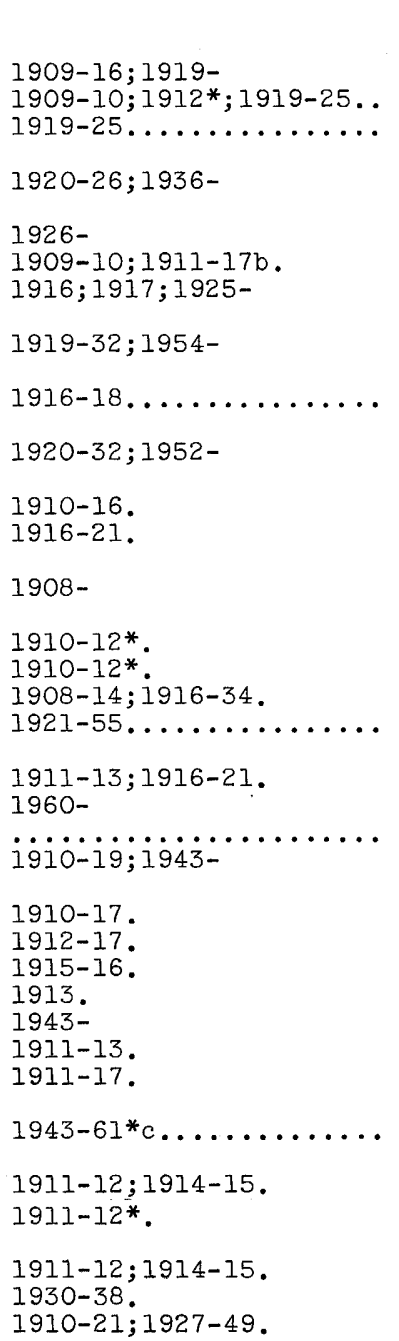 & 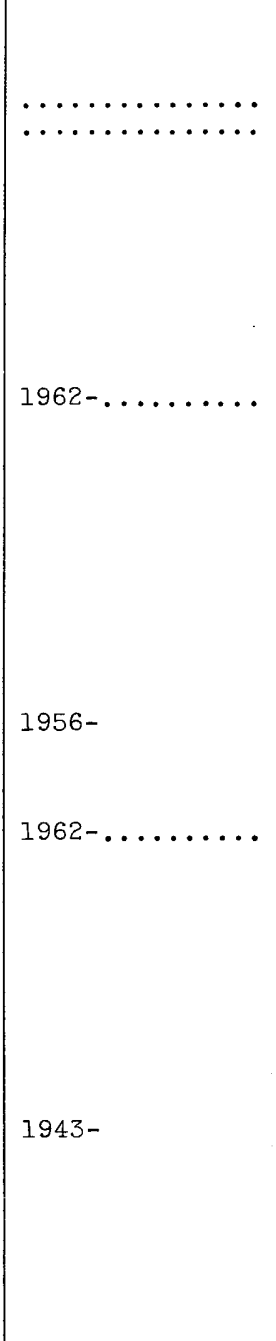 & $\begin{array}{l}1916 ; 1920-21 ; \\
\quad 1954-55 ; 1962-\end{array}$ \\
\hline
\end{tabular}

* Gage helghts, or gage helghts and discharge measurements only.

a Approximately.

c In files of district office. 


\begin{tabular}{|c|c|c|c|c|c|}
\hline \multirow[b]{2}{*}{ Station name } & \multirow{2}{*}{$\begin{array}{r}\text { Station } \\
\text { number }\end{array}$} & \multirow{2}{*}{$\begin{array}{c}\text { Drainage area } \\
\text { (sq mi) }\end{array}$} & \multicolumn{3}{|c|}{ Periods of record } \\
\hline & & & $\begin{array}{c}\text { Dally or monthly } \\
\text { flgures }\end{array}$ & Annual peaks & $\begin{array}{l}\text { Low-flow } \\
\text { measurements }\end{array}$ \\
\hline 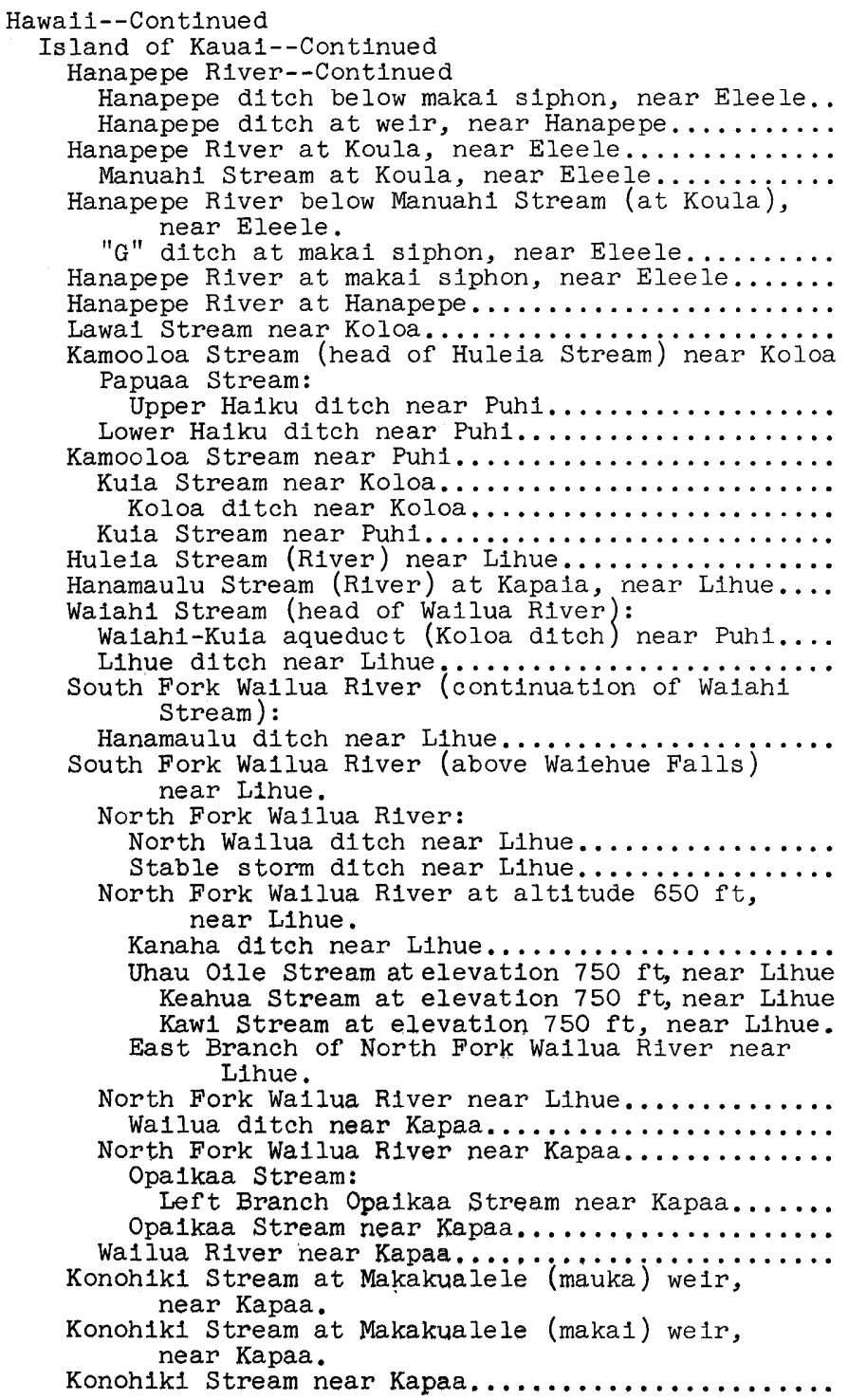 & $\begin{array}{l}450 \\
460 \\
470 \\
480 \\
490 \\
\\
500 \\
510 \\
520 \\
525 \\
530 \\
534 \\
536 \\
538 \\
540 \\
542 \\
545 \\
550 \\
560 \\
568 \\
570\end{array}$ & $\begin{array}{c}- \\
- \\
12.6 \\
5.44 \\
18.8 \\
- \\
20.5 \\
26.9 \\
6.60 \\
21.3 \\
- \\
- \\
5.94 \\
2.4 \\
- \\
5.04 \\
17.6 \\
6.41 \\
- \\
- \\
- \\
22.4\end{array}$ & 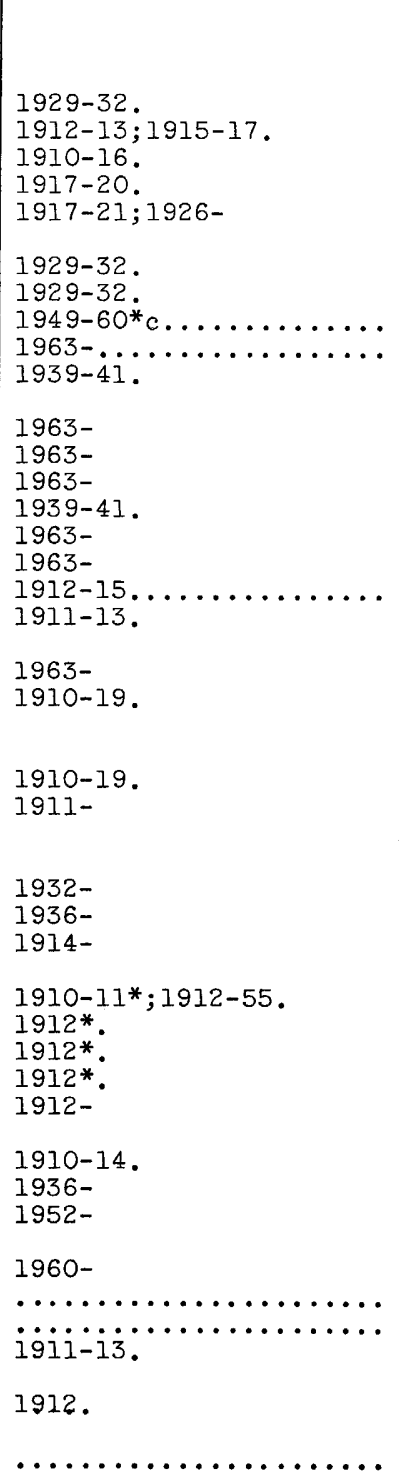 & $1962-$ & \\
\hline
\end{tabular}


North Fork Kaehulua Stream at Ka1nahola we1r, near Kapaa.

South Fork Kaehulua Stream at Walnamunamu weir, near Kapaa.

Kaehulua Stream at Kuhinoa weir, near Kapaa....... Kapaa Stream:

kaleha Stream

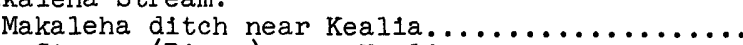

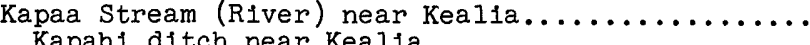

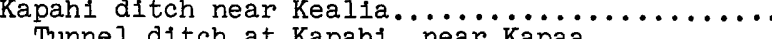
Tunnel ditch at Kapah1, near Kapaa.........

Kapaa.

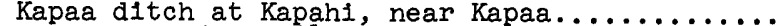

Kapaa Stream (River) at Kapah1 ditch intake, near Kapaa.

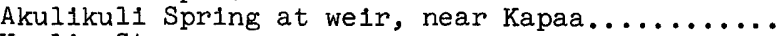
Kealia Stream:

Kaneha ditch (at Kaneha) (at weir) near Kealia Kapaa Stream at old highway crossing, near Kealia. Anahola River at elevation 1,140 ft, near Kealia.

Anahola ditch above wasteway (Kaneha Reservolr), near Kealla.

Anahola ditch wasteway near Kealia.......... Anahola ditch above Kaneha Reservoir, near Kealia.

Anahola Stream (River above dam at Kiokala) near Kealla.

Lower Anahola (Anahola) ditch at Klokala, near Kealia.

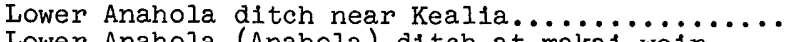

Lower Anahola (Anahola) ditch at makai weir, near Kealia. Anahola Stream (River above dam) at Kiokala Dam,
near Kealia.

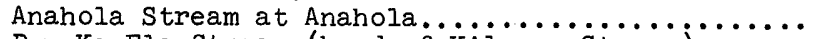

Puu Ka Ele Stream (head of Kilauea Stream):

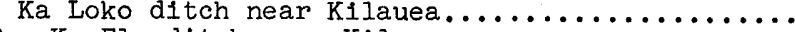

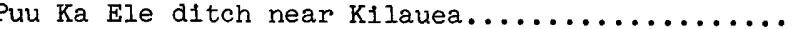

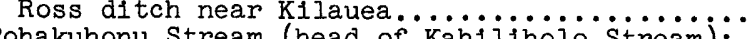

Kalihiwai ditch above wasteway, near Kilauea...

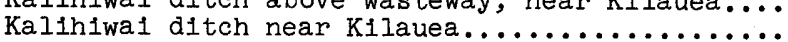

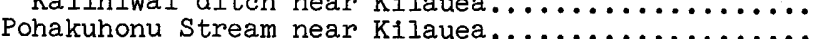

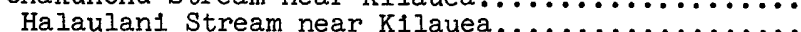
Halaulani Stream at altitude 400 fi. near

Halaulani Stream

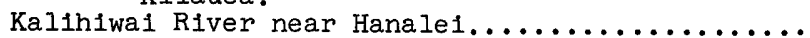

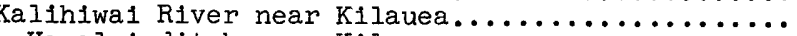

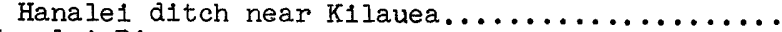

Hanale1 River:

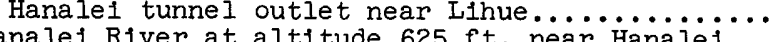

Hanalei River at altitude $625 \mathrm{ft}$, near Hanale1....

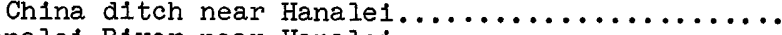

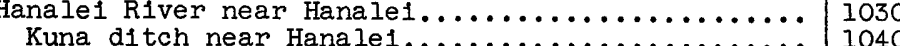

Gage heights,

a Approximately.

b Fragmentary

c In files of district office.

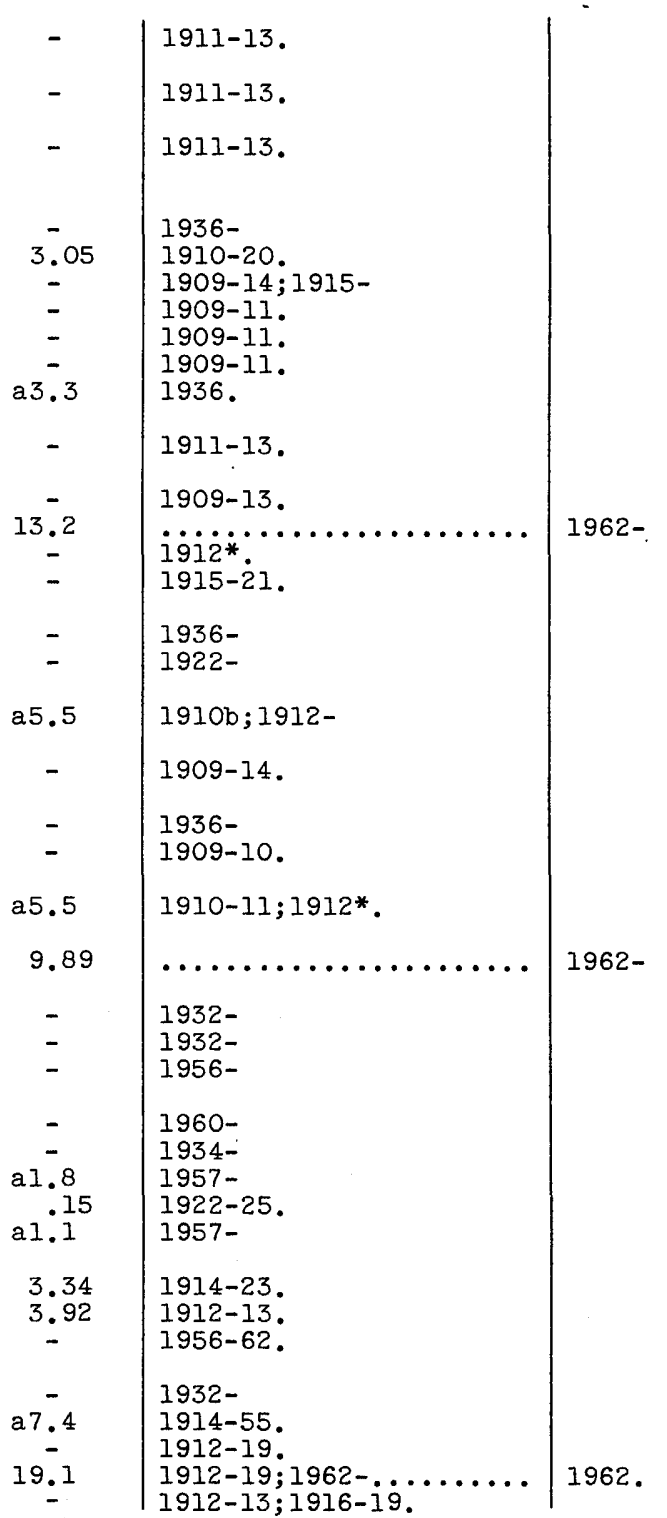




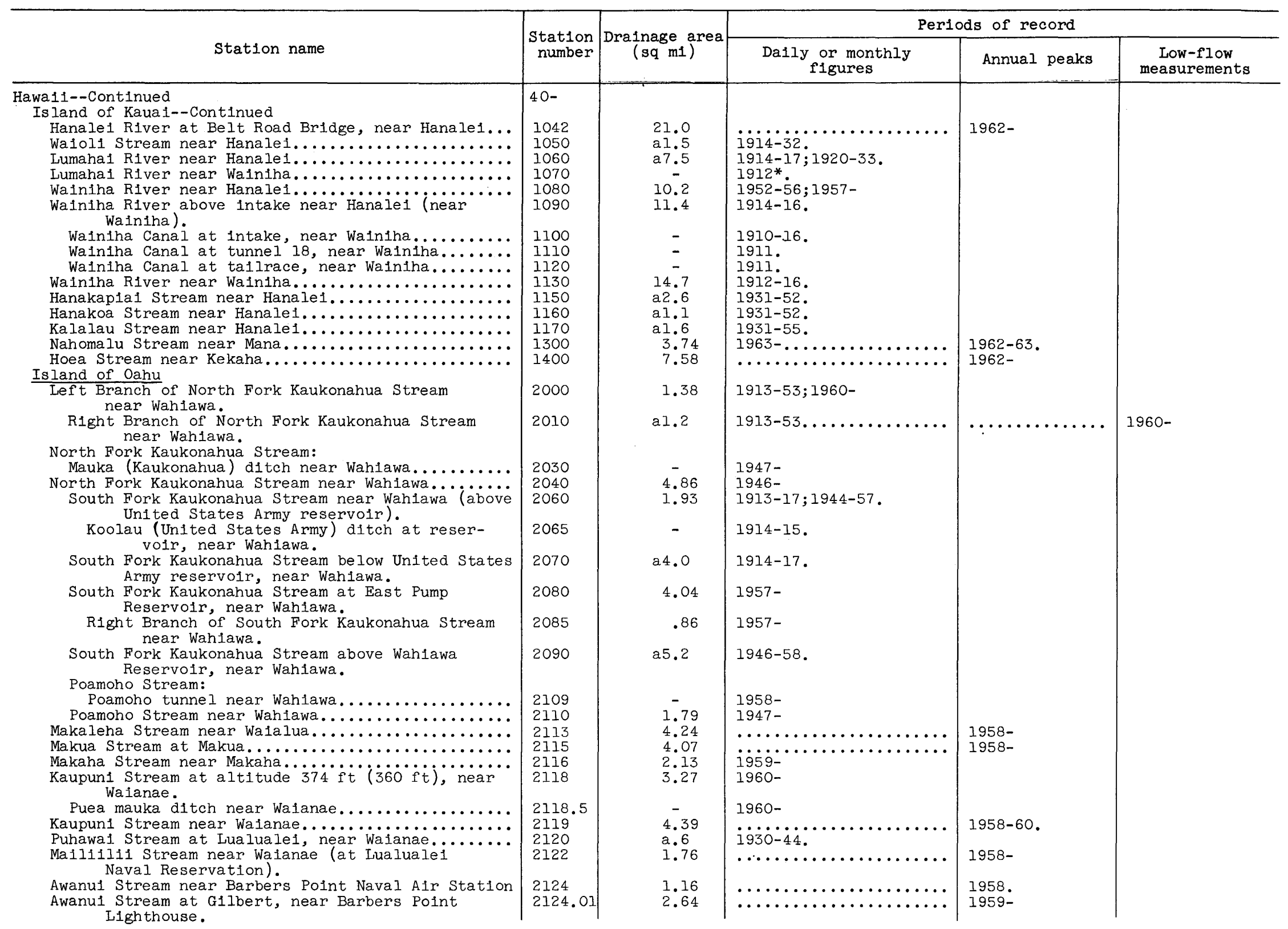


Drain "A" at Campbell Industrial Park, at Barbers Point Lighthouse.

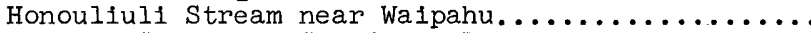

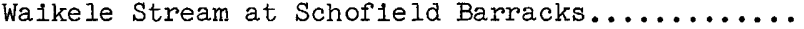
Walkele Stream at Wheeler Field.

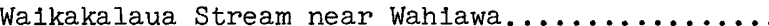

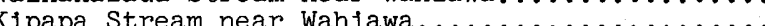

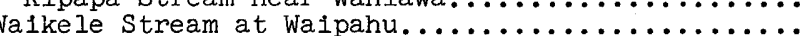
Pearl Harbor Springs at Waiawa, near Pearl city...

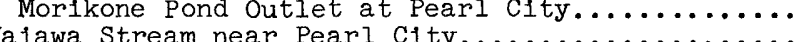
Waimano flood channel at Peari city.

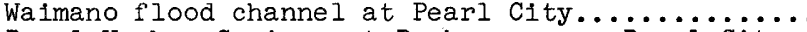
Pearl Harbor Springs at Puukapu, near Pearl City.

ings at Loko Kukona, nea Pearl City.

Pearl Harbor Springs at Kaluaoopu, near Pearl City Hawalian Electric Co. tunnel at Walau, near Pearl City.

Pearl Harbor Springs at Waiau, near Pearl City....

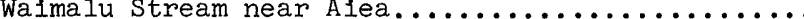
Pearl Harbor Springs at Kalauao, near Alea.......

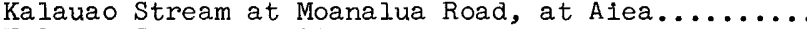

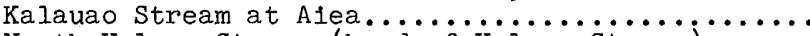
North Halawa Stream (head of Halawa Stream) near Aiea.

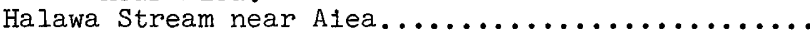

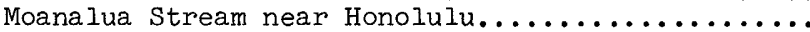
Moanalua Stream at altitude $100 \mathrm{ft}$, near Honolulu.

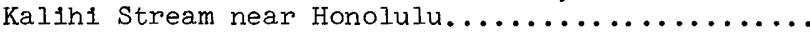

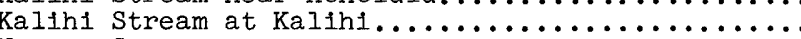
Lumu Stream:

ulumahu Stream:

Lulumahu ditch at upper Nuuanu Reservoir, near Honolulu.

(Nuuaru Stream at) Iuakaha weir in upper Nuuanu valley, near Honolulu.

Moole Stream:

Moole ditch, mauka station, near Honolulu...... 2315 Moole ditch, makai station, near Honolulu..... 2317

Nuuanu Stream below reservo1r 2 wasteway, near 2320 Honolulu.

Nuuanu Stream at Kuakini Street, near Honolulu.... 2350

Nuuanu Stream above Waolan1 Stream (confluence), at Honolulu.

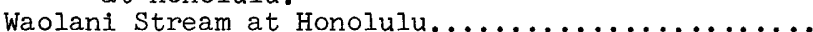

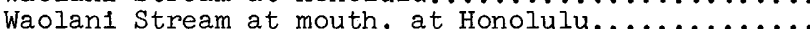
Pauoa Stream:

Kahuawai Spring near Honolulu (at upper Pauoa Valley).

Pauoa Stream at upper Pauoa Valley, near Honolulu Pauoa Stream at Lusitana Street, at Honolulu... east Branch Manoa Stream:

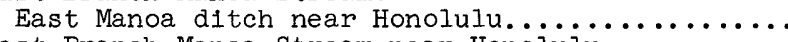

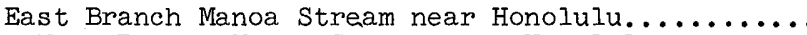

West Branch Manoa Stream near Honolulu...............

2350

2351

2354

2356

2360

2370

2375

2380

2390
2400

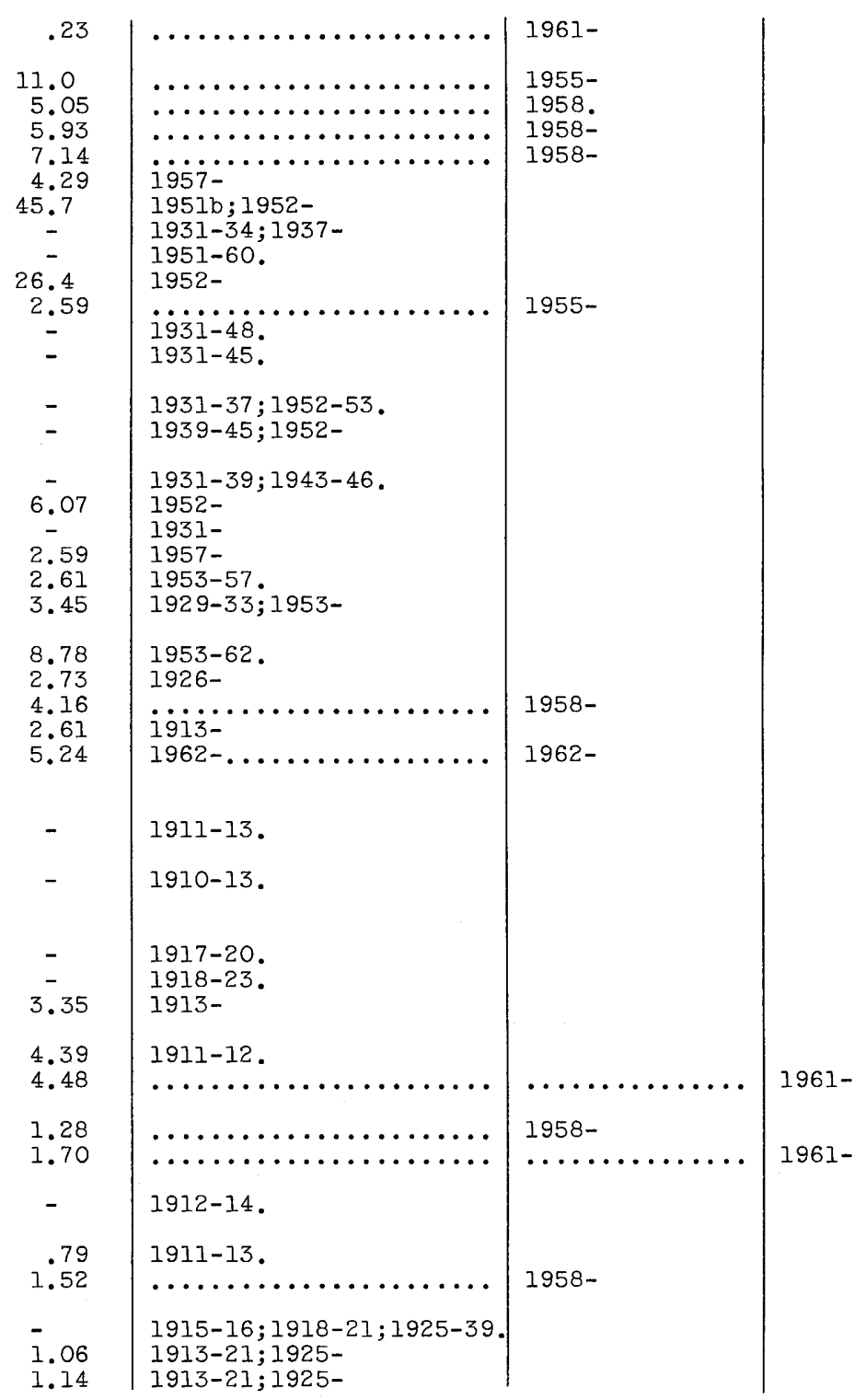

* Gage heights, or gage heights and discharge measurements only. 


\begin{tabular}{|c|c|c|c|c|c|}
\hline \multirow[b]{2}{*}{ Station name } & \multirow{2}{*}{$\begin{array}{r}\text { Station } \\
\text { number }\end{array}$} & \multirow{2}{*}{$\begin{array}{c}\text { Drainage area } \\
\text { ( } \mathrm{sq} \mathrm{mi})\end{array}$} & \multicolumn{3}{|c|}{ Periods of record } \\
\hline & & & $\begin{array}{c}\text { Daily or monthly } \\
\text { figures }\end{array}$ & Annual peaks & $\begin{array}{l}\text { Low-flow } \\
\text { measurements }\end{array}$ \\
\hline 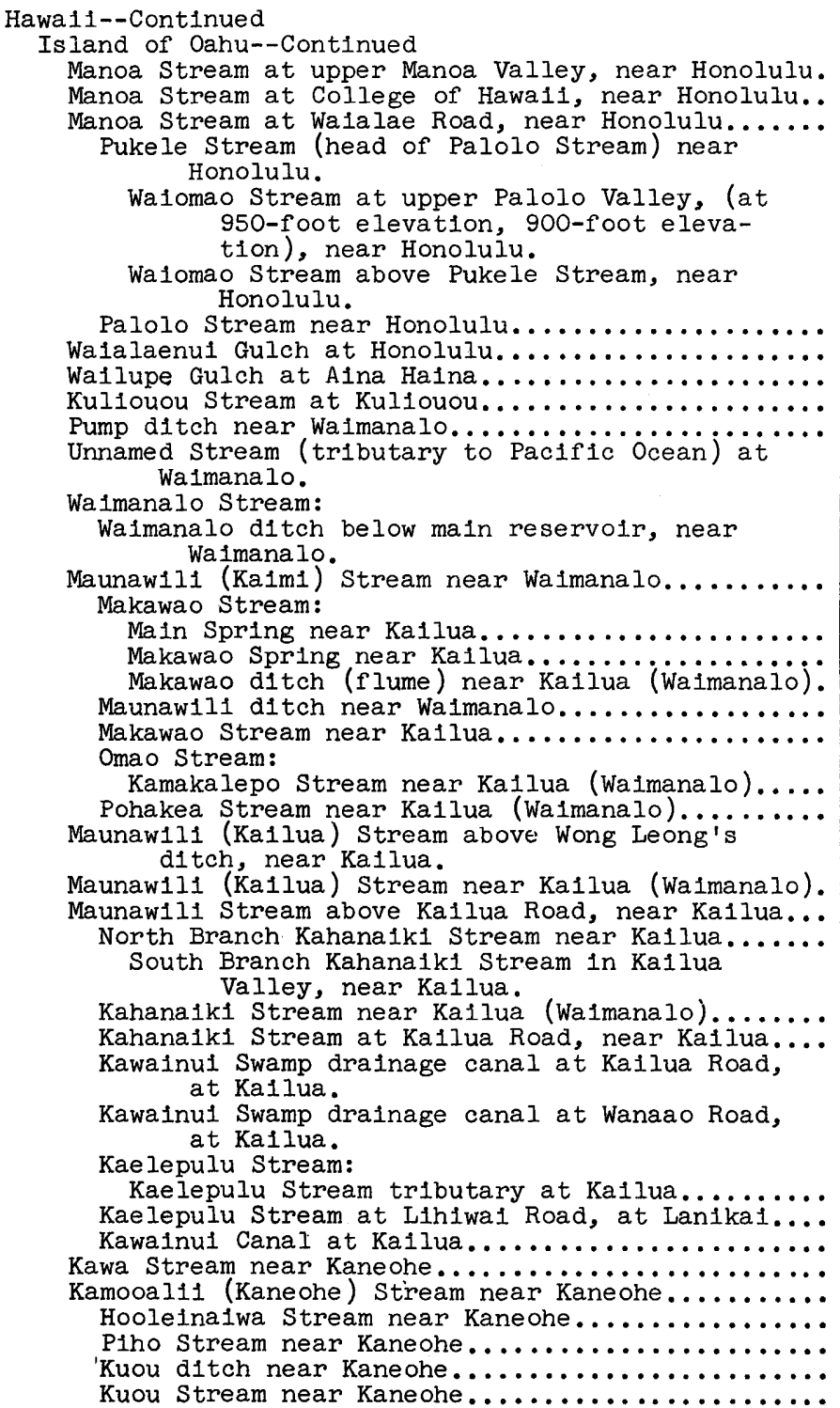 & $\begin{array}{l}2490 \\
2492 \\
2494 \\
2496 \\
2498 \\
2500 \\
2540 \\
\\
2560 \\
2570 \\
2580 \\
2600 \\
2605 \\
2610 \\
2620\end{array}$ & $\begin{array}{c}2.62 \\
4.99 \\
- \\
1.18 \\
.35 \\
1.04 \\
3.63 \\
1.75 \\
2.35 \\
1.21 \\
- \\
1.21 \\
\\
- \\
1.28 \\
- \\
- \\
- \\
2.04 \\
.82 \\
.21 \\
24.6 \\
24.6 \\
5.62 \\
.34 \\
- \\
.58 \\
1.45 \\
- \\
-\end{array}$ & 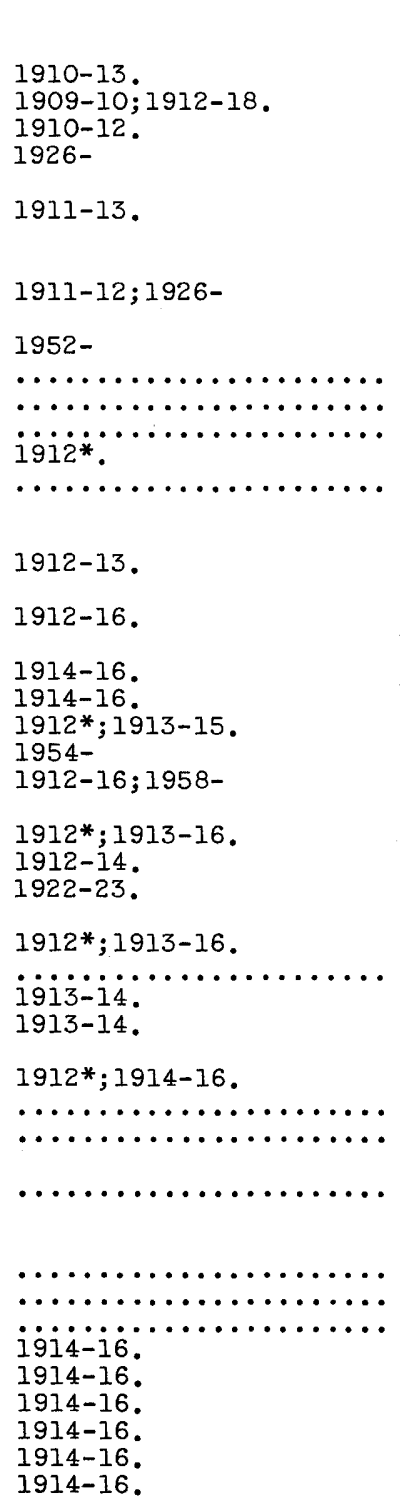 & $\begin{array}{l}\text { i962- } \\
1962- \\
\begin{array}{l}1963- \\
1962 . \\
1962-\end{array}\end{array}$ & $1960-$ \\
\hline
\end{tabular}


Luluku Stream at altitude $220 \mathrm{ft}(200 \mathrm{ft})$, near Kaneohe.

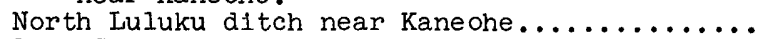

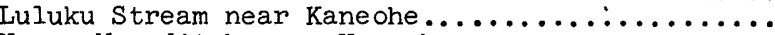

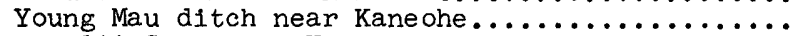

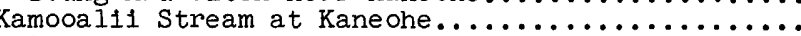
Ahlo ditch near Kaneohe...

Keaahala Stream at Kamehameha Highway, at Kaneohe

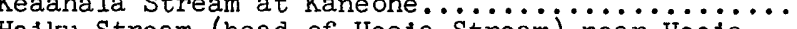
Halku Stream (head of Heela Stream) near Heela...

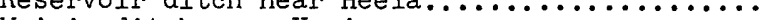
Waiplo ditch near Heela.

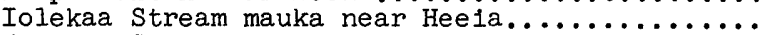

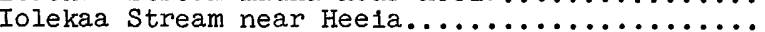

Hee1a Stream:

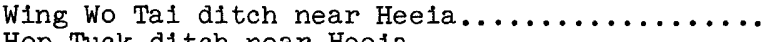

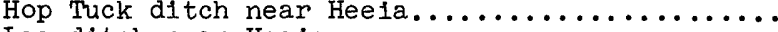

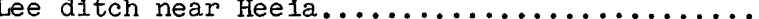
Kahaluu Stream:

Ahulmanu Stream near Kahaluu.

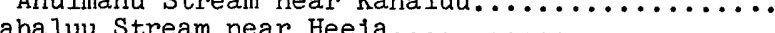
Kahaluu Stream left bank tributary near Kaholuu.

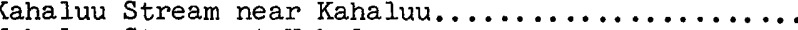

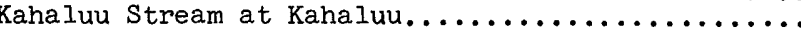
South Fork Wathee Stream near Heela.

North Fork Wathee Stream near Heela.

Waihee Stream at altitude $260 \mathrm{ft}(280 \mathrm{ft})$ near Heela.

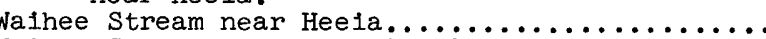
Walhee Stream above Kahaluu Stream, near Kahaluu Waiahole tunnel at Waianu, near walahole......... Walahole tunnel wasteway at intake 31 , near Waiahole.

Walahole tunnel at north portal, near Walahole... Walahole tunnel at adit 8 , near Wa1pahu...........

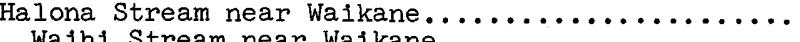
Walh1 Stream near Walkane............................. Walahole Stream at altitude $250 \mathrm{ft}$, near Walahole. Waiahole Stream near Waiahole (at Manianiaula, near Waikane).

Walanu Stream near Wa1kan

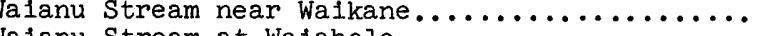

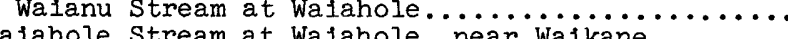
Walkane stream at Walahole, ${ }^{2}$. Walkane stream at altitude $75 \mathrm{ft}$, near Walkane....

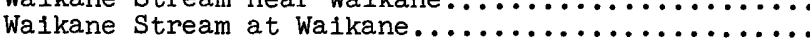
Waikane stream at Wa1kane.

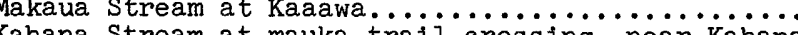
Kahana stream at mauka trall crossing, near Kahana

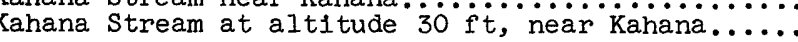
East Branch Kahana Stream near Kahana.......... Punaluu Stream near Hauula....................

Wa1hol Stream near Punaluu.
2709

2710

2730

2730

2740

2744.99

2745

2750

2760

2770

2780

2790

2800

2810

2820

2825

2831

2834

2835

2836

2838

2840

2845

2850

2860

2870

2872

2880

2890

2900

2920

2930

2940

2940

2949

2952

2959

2959.95

2960

2965

2970

2980

2990
* Gage heights, or gage helghts and discharge measurements only.

a Approximately.

d In files of Waiahole water Co.

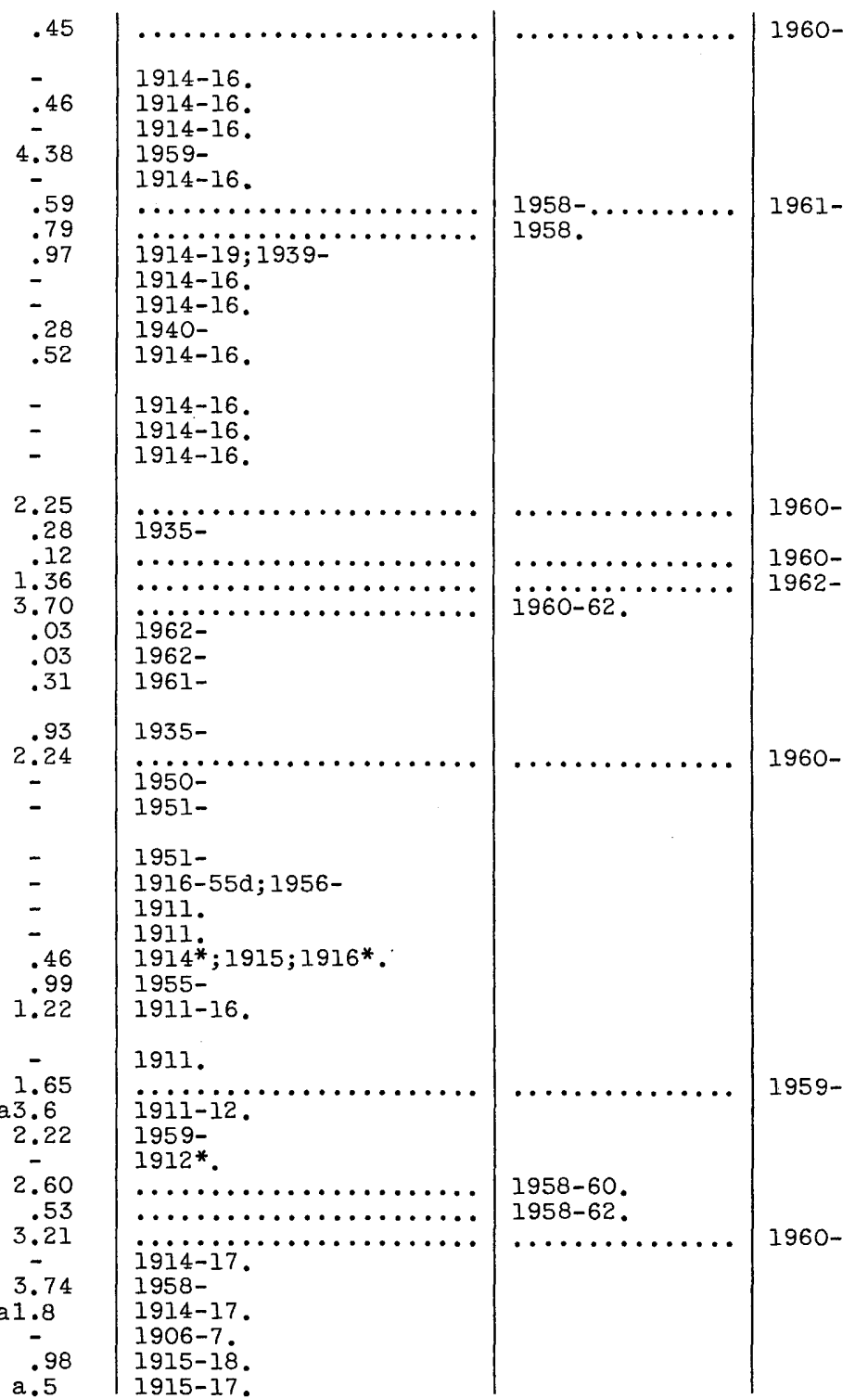

\begin{tabular}{l|l}
. .58 & $1915-18$. \\
$1915-17$.
\end{tabular} 


\begin{tabular}{|c|c|c|c|c|c|}
\hline \multirow[b]{2}{*}{ Station name } & \multirow{2}{*}{$\begin{array}{r}\text { Station } \\
\text { number }\end{array}$} & \multirow{2}{*}{$\begin{array}{c}\text { Drainage area } \\
(\mathrm{sq} \mathrm{m} \mathrm{l})\end{array}$} & \multicolumn{3}{|c|}{ Periods of record } \\
\hline & & & $\begin{array}{c}\text { Daily or monthly } \\
\text { figures }\end{array}$ & Annual peaks & $\begin{array}{c}\text { Low-flow } \\
\text { measurements }\end{array}$ \\
\hline 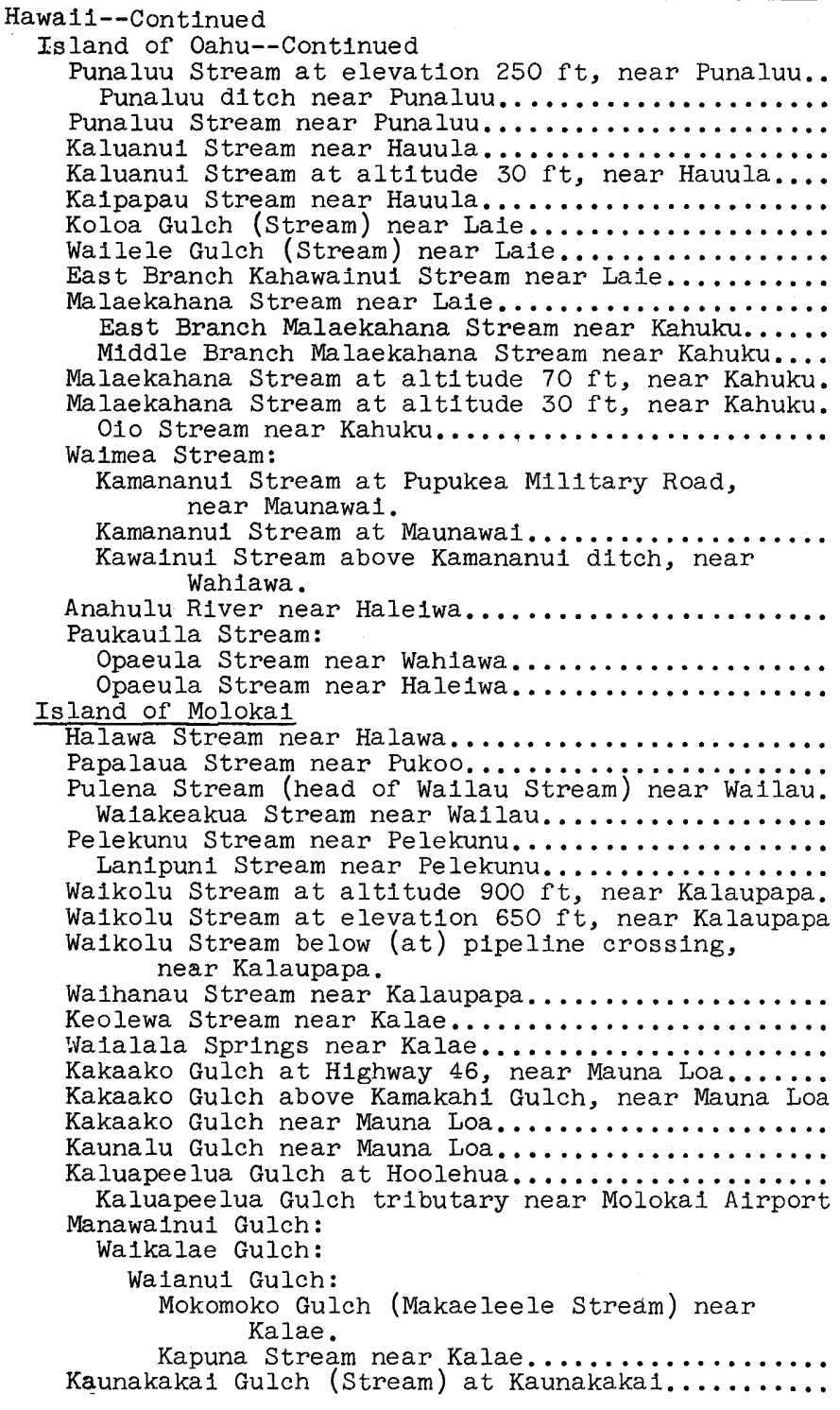 & \begin{tabular}{|l|}
$40-$ \\
3010 \\
3020 \\
3030 \\
3040 \\
3045 \\
3050 \\
3060 \\
3070 \\
3080 \\
3089.9 \\
3090 \\
3100 \\
3105 \\
3105.01 \\
3110 \\
\\
3250 \\
\\
3300 \\
3350 \\
\\
3400 \\
\\
3450 \\
3500 \\
4000 \\
4010 \\
4020 \\
4030 \\
4040 \\
4050 \\
4055 \\
4060 \\
4080 \\
4090 \\
4100 \\
4110 \\
4113 \\
4113.2 \\
4114 \\
4116 \\
4118 \\
4119 \\
\end{tabular} & $\begin{array}{c}2.78 \\
-. \\
2.78 \\
a .5 \\
2.12 \\
-. \\
2.9 \\
a .5 \\
.53 \\
.64 \\
1.66 \\
.69 \\
2.94 \\
3.80 \\
2.22 \\
3.13 \\
9.79 \\
4.74 \\
11.9 \\
2.98 \\
5.96 \\
4.62 \\
a 2.0 \\
4.38 \\
1.41 \\
2.62 \\
1.09 \\
1.99 \\
2.99 \\
3.68 \\
1.18 \\
.18 \\
.5 \\
1.45 \\
5.34 \\
.28 \\
1.46 \\
1.56\end{array}$ & 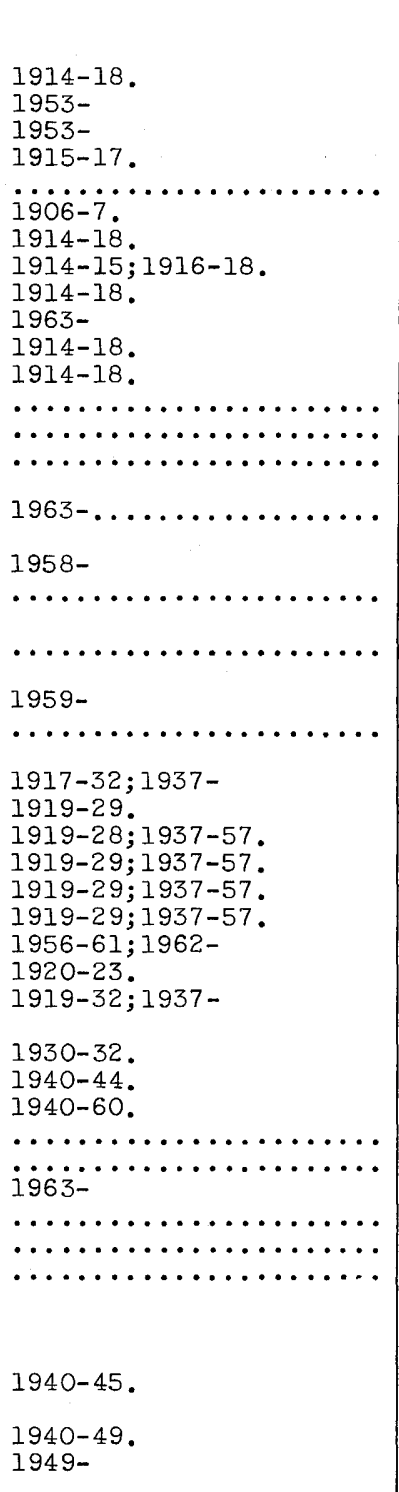 & $\begin{array}{l}1958 . \\
1959- \\
1958- \\
\ldots \ldots \ldots \ldots \ldots \ldots \\
\ldots \ldots \ldots \ldots \ldots \ldots \\
1958- \\
1958-\end{array}$ & $1960-63$ \\
\hline
\end{tabular}


Kawela Gulch:

(Rlght Branch of) East Fork Kawela Gulch Stream) near Kamalo. sion dam (Oheo Stream at elevation 1,550 $\mathrm{f}^{\prime} \mathrm{t}$, below diversion dam), near Kipahulu.

Hahalawe Gulch (Right Branch Hahalawe Stream, Kahalawe Stream) near Kipahulu.

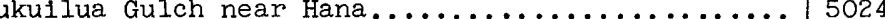

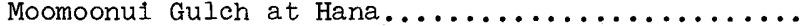
Hana flume:

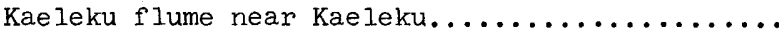

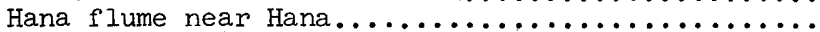
Makapip1 Stream:

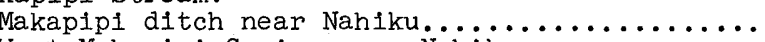

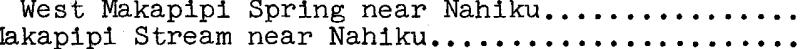

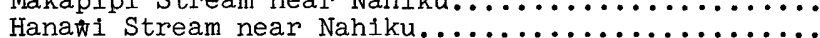
Hanawi Stream below Government Road, near Nahiku..

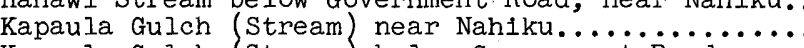
Kapaula Gulch (Stream) below Government Roàd, near Nahiku.

Koolau ditch at Nahiku weir, near Nahiku..........

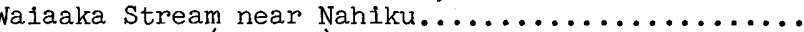

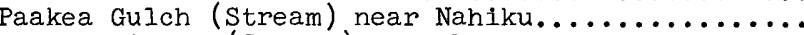

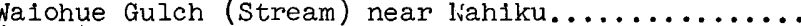

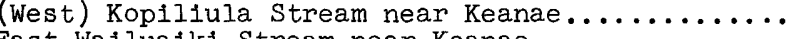

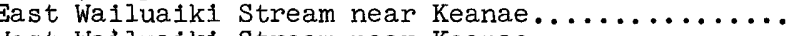

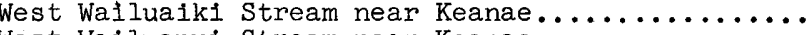

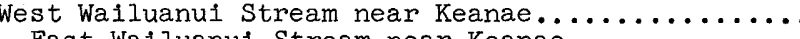

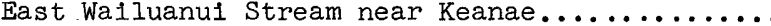

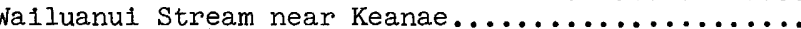
Palauhulu Stream:

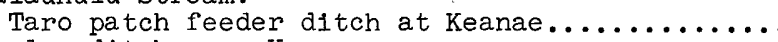

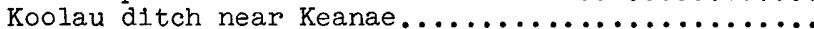
Honomanu Stream at Ha1ku-uka boundary, near Kaililil.

Seventh Branch Honomanu Stream at Ha1ku-uka boundary, near Kailiili.

Fourth Branch Honomanu Stream at Haiku-uka boundary, near Kaililli.

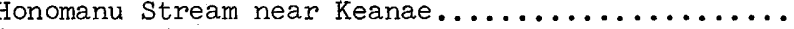

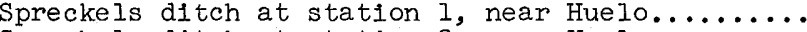
Spreckels ditch at station 2 , near Huelo...........

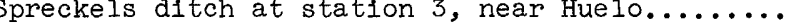
Haipuaena Stream:

Kula diversion from Haipuaena Stream, near olinda.$$
5310
$$

\begin{tabular}{|c|c|c|}
\hline .45 & 1946- & \\
\hline $\begin{array}{r}2.12 \\
.24\end{array}$ & $\ddot{i g} \dot{9} \ddot{7}_{-} \cdots \cdots \cdots \cdots \cdots \cdots \cdots$ & $1963-$ \\
\hline $\begin{array}{l}.48 \\
.94\end{array}$ & $i \ddot{9} 63-\ldots \ldots \ldots \ldots \ldots$ & 1963- \\
\hline $\begin{array}{l}1.91 \\
.76 \\
6.29\end{array}$ & 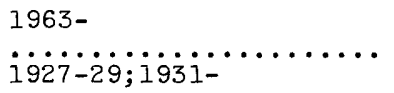 & 1963- \\
\hline .43 & $1927-$ & \\
\hline $\begin{array}{l}.48 \\
.90\end{array}$ & $\cdots \cdots \cdots \cdots \cdots \cdots \cdots \cdots \cdots \cdots \cdots \cdots \cdots$ & $\begin{array}{l}1963- \\
1963-\end{array}$ \\
\hline- & $\begin{array}{l}1940-45 \\
1940-45\end{array}$ & \\
\hline $\begin{array}{l}- \\
- \\
1.93 \\
3.49 \\
5.03 \\
.69 \\
.93\end{array}$ & $\begin{array}{l}1948- \\
1932-45 . \\
1932-45 \\
1914-16 ; 1921- \\
1932-47 \\
1921-63 \\
1932-47\end{array}$ & \\
\hline $\begin{array}{l}- \\
.10 \\
.34 \\
.32 \\
4.31 \\
3.11 \\
3.66 \\
1.93 \\
.51 \\
2.51\end{array}$ & $\begin{array}{l}1919- \\
1932-47 . \\
1932-47 \\
1921-63 ; \\
1914-17 ; 1921- \\
1913-17 ; 1921^{*} ; 1922-58 . \\
1914-17 ; 1921- \\
1913-17 ; 1922-57 . \\
1914-17 ; 1921-58 . \\
1932-36 ; 1938-47 .\end{array}$ & \\
\hline $\begin{array}{l}- \\
- \\
2.54\end{array}$ & $\begin{array}{l}1934- \\
1910-12 ; 1917- \\
1919-27 ; 1932-34 ; 1962-\end{array}$ & \\
\hline a. 3 & $1932-33$ & \\
\hline a. 1 & $1932-33$ & \\
\hline $\begin{array}{l}3.17 \\
- \\
- \\
-\end{array}$ & $\begin{array}{l}1913- \\
1910-13 . \\
1911-13 . \\
1910-13 .\end{array}$ & \\
\hline - & $1945-$ & \\
\hline
\end{tabular}

* Gage heights, or gage heights and discharge measurements only.

a Approximately. 


\begin{tabular}{|c|c|c|c|c|c|}
\hline \multirow[b]{2}{*}{ Station name } & \multirow{2}{*}{$\begin{array}{r}\text { Station } \\
\text { number }\end{array}$} & \multirow{2}{*}{$\begin{array}{c}\text { Drainage area } \\
(\mathrm{sq} \mathrm{mI})\end{array}$} & \multicolumn{3}{|c|}{ Periods of record } \\
\hline & & & $\begin{array}{c}\text { Dally or monthly } \\
\text { flgures }\end{array}$ & Annual peaks & $\begin{array}{l}\text { Low-flow } \\
\text { measurements }\end{array}$ \\
\hline 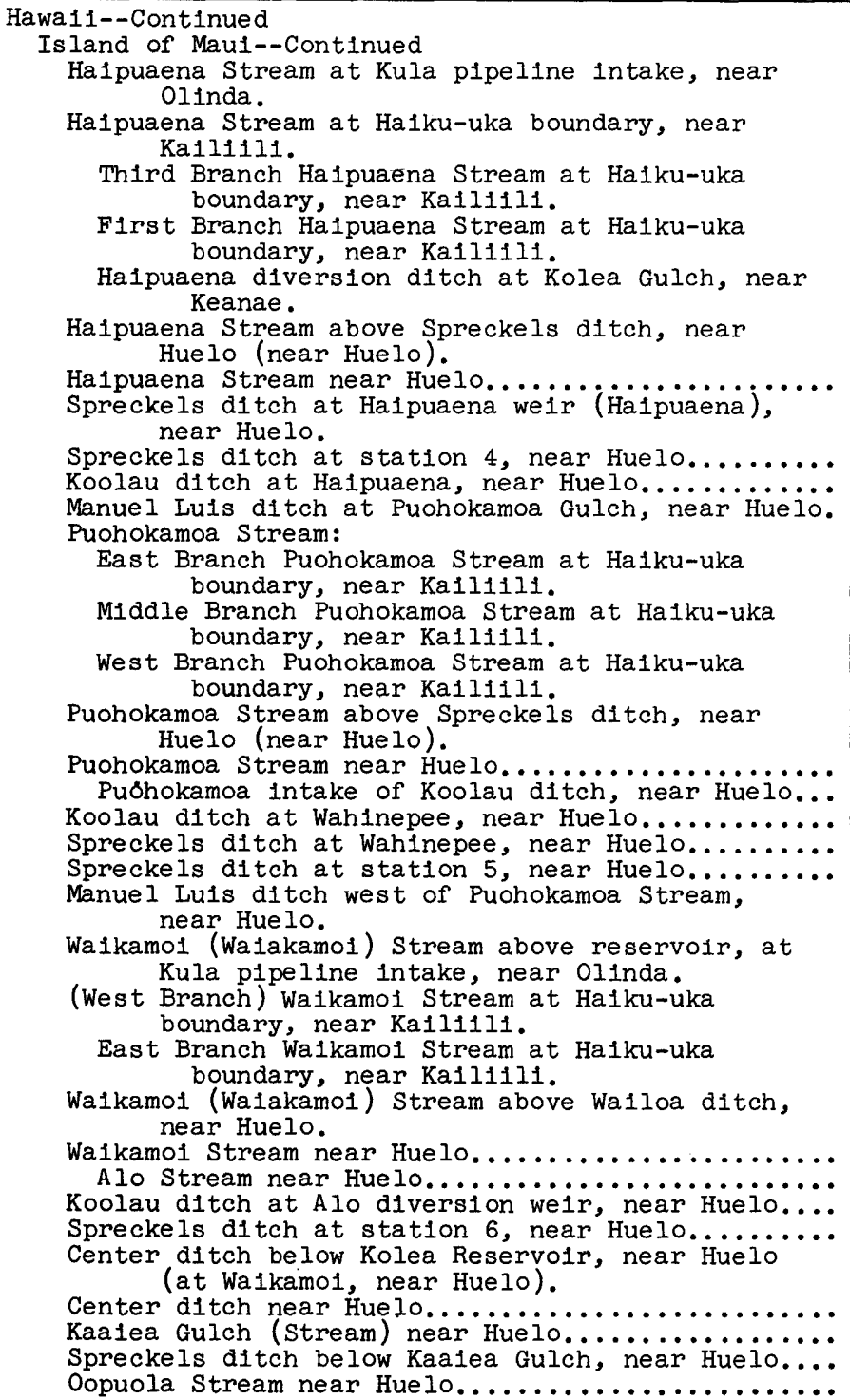 & 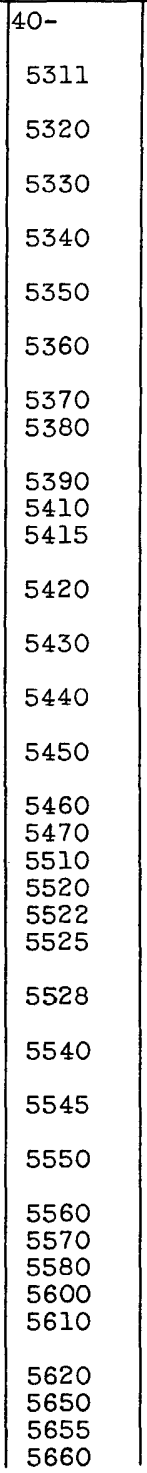 & $\begin{array}{c}0.26 \\
.61 \\
.06 \\
.05 \\
- \\
1.16 \\
\text { a1.1 } \\
- \\
- \\
- \\
.14 \\
.48 \\
.50 \\
2.35 \\
\text { a2.6 } \\
- \\
- \\
- \\
- \\
2.50 \\
3.46 \\
.07 \\
3.98 \\
-47 \\
- \\
-\end{array}$ & $\begin{array}{l}1946 b ; 1947- \\
1919-27 ; 1932-34 \ldots \ldots \ldots \\
1932-33 . \\
1932-33 . \\
1938-60 . \\
1913- \\
1910-13 . \\
1922- \\
1910-13 . \\
1932- \\
1917- \\
1919-27 ; 1932-33 \ldots \ldots \ldots \\
1919-27 ; 1932-34 ; 1962- \\
1919-28 ; 1932-34 \ldots \ldots \ldots \\
1913- \\
1910-13 . \\
1922-30 . \\
1922-29 . \\
1928-38 . \\
1911-13 . \\
1930-35 . \\
1953- \\
1918-28 ; 1932-34 \ldots \ldots \ldots \\
1918-28 ; 1932-33 \ldots \ldots \ldots \\
1922-57 . \\
1910-22 . \\
1910-57 . \\
1908-11 . \\
1911-13 . \\
1918-30 . \\
1910-12 . \\
1921-62 . \\
1917-30 . \\
1930-57 .\end{array}$ & $\begin{array}{c}\cdots \cdots \cdots \cdots \\
\cdots \ldots \ldots \ldots \ldots \ldots\end{array}$ & $\begin{array}{l}1963- \\
1962-\end{array}$ \\
\hline
\end{tabular}


Oopuola Stream above Spreckels ditch crossing, near Huelo (near Huelo).

Spreckels ditch at station 7 , near Huelo........................

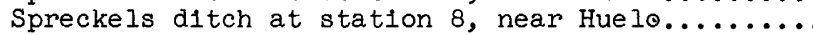
Nallilithaele Stream:

Second Branch Nallilihaele Stream at Haikuuka boundary, near Kallilli.

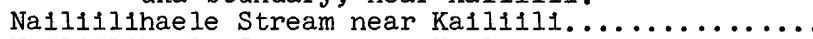
Na1l11lhaele stream near huelo............. Nallilihaele Stream below New Hamakua ditch, near Huelo.

New Hamakua ditch at Nailiflihaele weir, near Huelo New Hamakua ditch at station No. 1, near Huelo... Kailua Stream at Haiku-uka boundary, near Ka1lilil

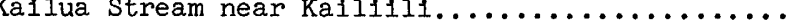
Tenth Branch Kallua Stream at Haiku-uka boundary, near Kallilli.

Ninth Branch Ka1lua Stream at Ha1ku-uka boundary, near Kaili1li.

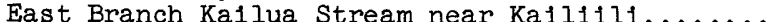

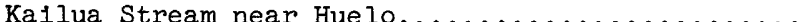
New Hamakua ditch at station No, 2 , near Huelo.... New Hamakua ditch at station No. 3, near Huelo... New Hamakua ditch at station No. 4, near Huelo.... Kailua Stream:

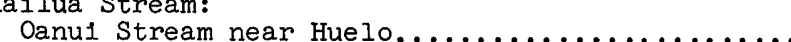

New Hamakua ditch at station No. 5 , near Huelo....

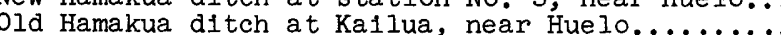
Kallua Stream near Huelo (station is downstream from New Hamakua ditch crossing).

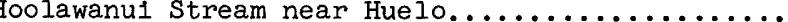

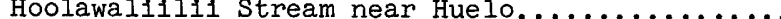

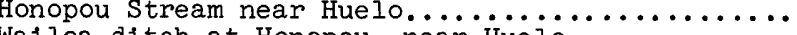
Wa1loa ditch at Honopou, near Huelo $\ldots \ldots \ldots \ldots \ldots$ New Hamakua ditch at Honopou, near Huei........... Honopou Stream at Lowrie ditch siphon, near Hueio. Lowrle ditch at Honopou Gulch (Opana weir), near Huelo.

Honopou Stream above Ha1ku ditch, near Huelo...... Haiku ditch at Honopou Gulch (at Peahi weir, at Manawai Gulch, at Kapalalaea Gulch), nea Kailua.

Honopou Stream below Ha1ku ditch, near Huelo...... New Hamakua ditch at Halehaku weir, near Huelo... Halehaku Gulch weir at New Hamakua ditch, near Hue 10.

Halehaku Guich (East Branch Opana Stream) near Huelo.

East Branch Opana Gulch (Stream) at Ha1ku-uka boundary, near Kaililli.

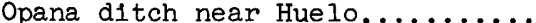

Opana Stream near Huelo.

Kauhikoa ditch (Old Hamakua ditch) at Opana weir, near Huelo.

Kaluanui ditch at Puuomalei, near Hamakuapoko..... 6030

* Gage helghts, or gage helghts and discharge measurements only.

approximately.

\begin{tabular}{|c|c|c|c|}
\hline .58 & $1910-15$ & & \\
\hline - & $1911-12$. & & \\
\hline- & $1911-13$ & & \\
\hline a. 2 & $1932-33$ & & \\
\hline $\begin{array}{l}.11 \\
3.58 \\
23.6\end{array}$ & 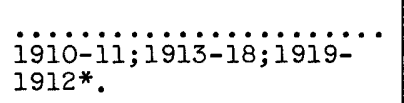 & $\cdots \cdots \cdots \cdots \cdots$ & $1963-$ \\
\hline $\begin{array}{l}- \\
- \\
\text { a.8 } \\
1.01 \\
\text { a.1 }\end{array}$ & $\begin{array}{l}1910-12 \\
1912^{*} \\
1918-28 ; 1932-34 \\
1963- \\
1932-33\end{array}$ & & \\
\hline a. 2 & $1932-33$ & & \\
\hline $\begin{array}{l}.35 \\
2.41 \\
- \\
-\end{array}$ & 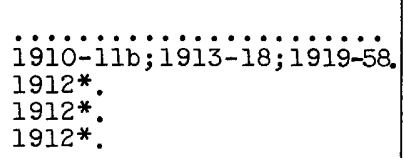 & $\ldots \ldots \ldots \ldots \ldots$ & $1963-$ \\
\hline $\begin{array}{l}a .9 \\
= \\
-\end{array}$ & $\begin{array}{l}1910-11 ; 1913-16 \\
1912^{*} \\
1919-\dot{2} 2 \\
1912^{*}\end{array}$ & & \\
\hline $\begin{array}{l}1.34 \\
.55 \\
.64 \\
- \\
- \\
- \\
2.0\end{array}$ & $\begin{array}{l}1910- \\
1912-57 . \\
1910- \\
1922- \\
1918- \\
1918-22 ; 1936- \\
1932-47 \text {. } \\
1910-27 ; 1930-\end{array}$ & & \\
\hline 2.2 & $\begin{array}{l}1932-47 \\
1910-28 ; 1930-\end{array}$ & & \\
\hline$\frac{2.3}{-}$ & $\begin{array}{l}1932-47 \\
1910-23 \\
1910-12\end{array}$ & & \\
\hline al. 4 & $1911 ; 1912 *$. & & \\
\hline a. 6 & $1932-33$ & & \\
\hline $\begin{array}{l}- \\
-\end{array}$ & $\begin{array}{l}1910-12 \\
1910-12 * \\
1910-28\end{array}$ & & \\
\hline- & $1910-12$ & & \\
\hline
\end{tabular}







Kauaula Stream at mouth, near Lahaina........... 6433

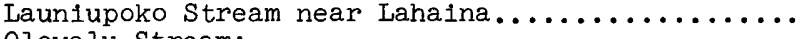

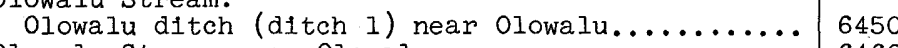

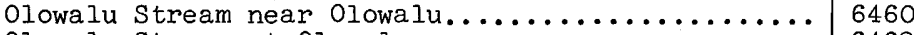

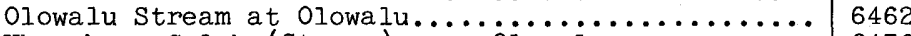

Ukumehame Gulch (Stream) near Olowalu............ 6470

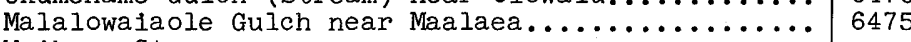

Waikapu Stream:

South Side Waikapu ditch near Waikapu.......... 6480

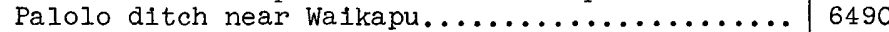

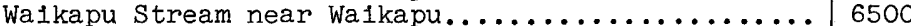

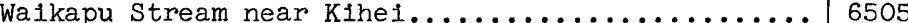

Wa1akoa Gulch:

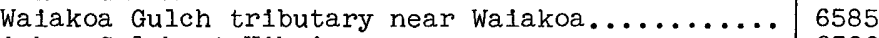

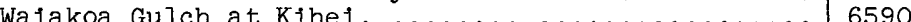

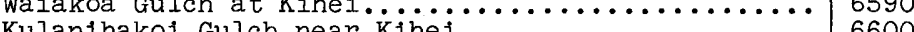

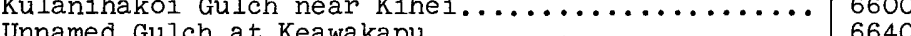

Is land of Hawa11

Walakea Stream (at middle flume house) near Mountain View.

Olaa flume at Kaumana, near Hilo...................... 7010

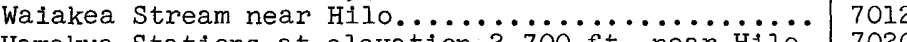

Hamakua Stations at elevation 2,700 ft, near Hilo. 7020

Walluku River at Pukamaul, near Hilo........... 7030

Walluku River above Hilo Boarding School ditch intake, near Hilo.

H1lo Boarding School ditch at intake, near H1lo.

Hilo Boarding School ditch near Hilo........... Kapehu Stream:

Kapehu ditch:

Kapehu ditch diversion near Hilo.......... 7070

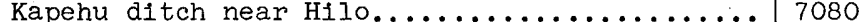

Kapehu Stream at Pilhonua, near Hilo............ 7090

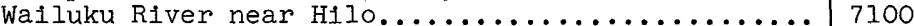

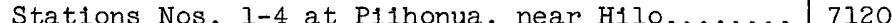

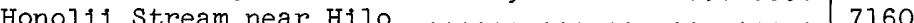

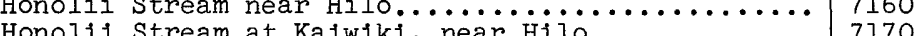

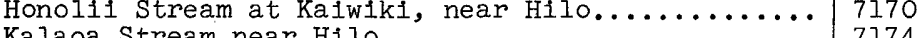

Kala stran

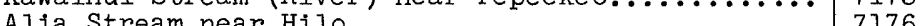

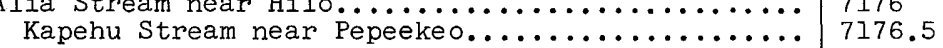

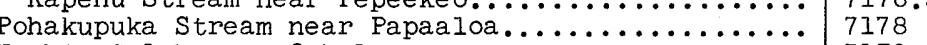

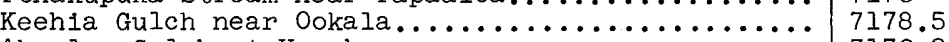

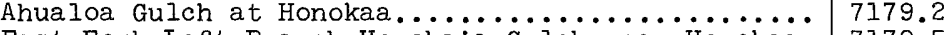

East Fork Left Branch Honokaia Gulch near Honokaa.

Waipio River:

Kawainul Stream at elevation 2,120 ft, near Waipio.

Branch 3 Kawainui Stream at elevation 1,700 ft,

Kawainu1 Stream at elevation 1,435 ft, near Waipio.

Branch 2 Kawainu1 Stream at elevation 1,405 ft, near Waipio.

* Gage heights, or gage heights and discharge measurements only. a Approximately.

e Combined flow of stream and ditch below point of diversion.

f Approximately, $79 \mathrm{sq} \mathrm{mi}$ probably noncontributing.

\begin{tabular}{|c|c|c|}
\hline $\begin{array}{l}4.30 \\
1.13\end{array}$ & $\ddot{i} \ddot{i} \ddot{i-i} \ddot{7}_{.} \cdots \ldots \ldots \ldots \ldots$ & 1963- \\
\hline $\begin{array}{r}- \\
4.00 \\
4.08 \\
3.75 \\
.64\end{array}$ & $\begin{array}{l}1911- \\
1913-16 . \\
1963- \\
1911 ; 1912 * ; 1913-19 . \\
\ldots \ldots \ldots \ldots \ldots \ldots \ldots \ldots \ldots\end{array}$ & $1963-$ \\
\hline $\begin{array}{l}- \\
2.76\end{array}$ & $\begin{array}{l}1910-17 \\
1910-17 \\
1910-17\end{array}$ & \\
\hline 6.97 & $\ldots \ldots \ldots \ldots \ldots \ldots \ldots$ & 1963- \\
\hline $\begin{array}{l}10.98 \\
14.1 \\
3.85\end{array}$ & 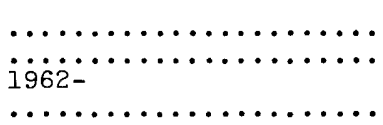 & $\begin{array}{l}1963- \\
1963- \\
1963-\end{array}$ \\
\hline- & 1930- & \\
\hline${ }^{9-}{ }^{-}$ & $\begin{array}{l}1917-20 . \\
1957- \\
1911-13 \\
1923-28 ; 1929-40 . \\
1928-\end{array}$ & \\
\hline$\overline{-}$ & $\begin{array}{l}1931-40 \\
1918-19\end{array}$ & \\
\hline $\begin{array}{c}- \\
\overline{4.84} \\
15 \\
- \\
28.0 \\
12.2\end{array}$ & $\begin{array}{l}1954-62 . \\
1938-62 \\
1928-37 \\
1911-13 ; 1918-19 \\
1912 * \\
1924-32 \\
1911-13\end{array}$ & \\
\hline $\begin{array}{l}.08 \\
a 9.2 \\
.58\end{array}$ & $\begin{array}{l}19 i 2 \ldots \ldots \ldots \ldots \ldots \ldots \ldots \ldots \ldots \\
1962-\end{array}$ & $1962-$ \\
\hline $\begin{array}{l}1.09 \\
2.94\end{array}$ & $i \ddot{q} 62 . \ldots \ldots \ldots \ldots \ldots$ & $1962-$ \\
\hline $\begin{array}{l}.31 \\
1.79 \\
5.67\end{array}$ & 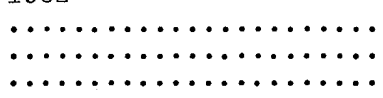 & $\begin{array}{l}1962- \\
1962- \\
1962-\end{array}$ \\
\hline - & $1901-2$ & \\
\hline- & $1901-2$ & \\
\hline- & $1901-2$ & \\
\hline - & $1901-2$ & \\
\hline
\end{tabular}




\begin{tabular}{|c|c|c|c|c|c|}
\hline \multirow[b]{2}{*}{ Station name } & \multirow{2}{*}{$\begin{array}{r}\text { Station } \\
\text { number }\end{array}$} & \multirow{2}{*}{$\begin{array}{c}\text { Drainage area } \\
(\mathrm{sq} \mathrm{m} \mathrm{l})\end{array}$} & \multicolumn{3}{|c|}{ Perlods of record } \\
\hline & & & $\begin{array}{c}\text { Dally or monthly } \\
\text { flgures }\end{array}$ & Annual peaks & $\begin{array}{l}\text { Low-flow } \\
\text { measurements }\end{array}$ \\
\hline 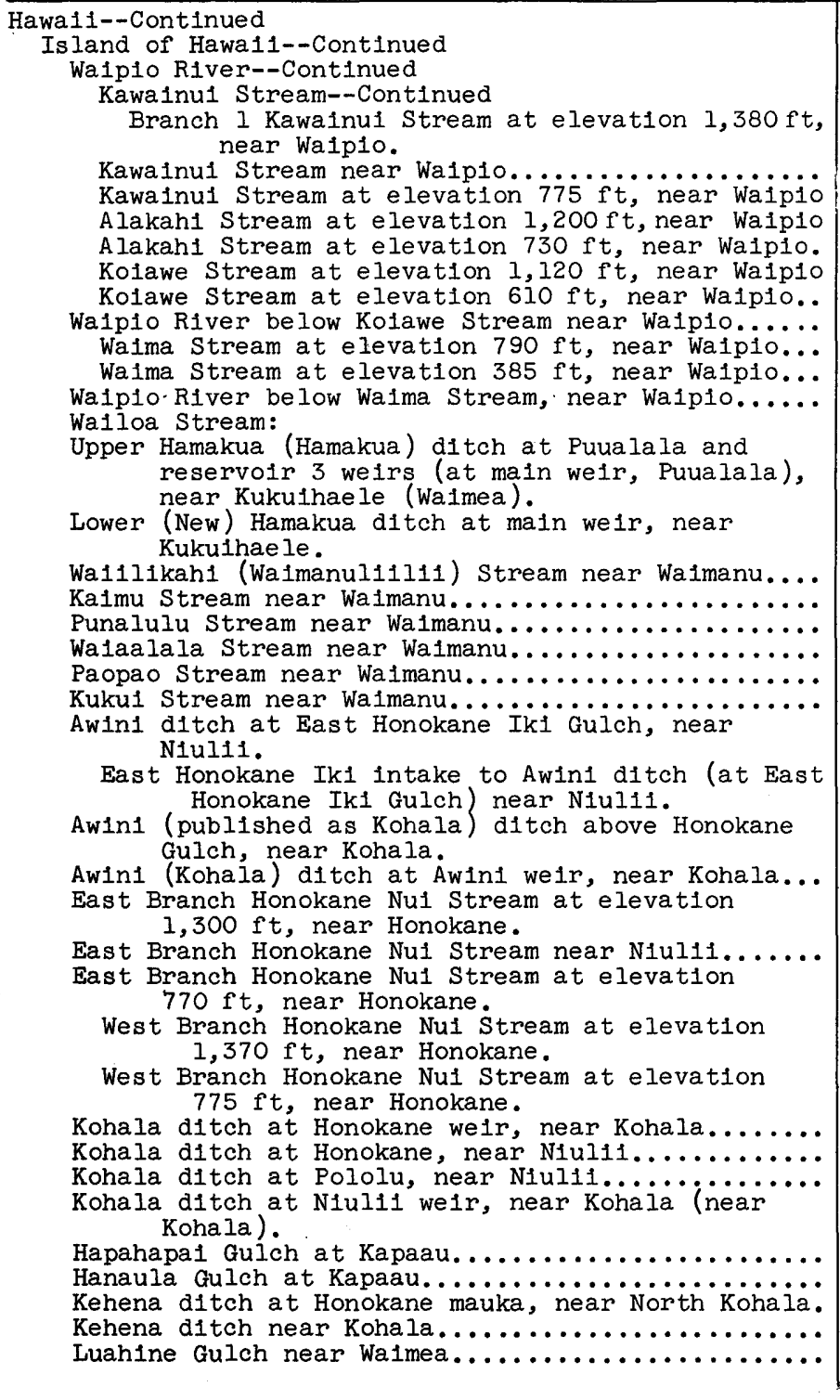 & $\begin{array}{l}7226 \\
7230 \\
7240 \\
7280 \\
7290 \\
7300 \\
7310 \\
7320 \\
7321 \\
7321.5 \\
7322 \\
7323 \\
\\
7329 \\
7370 \\
7380 \\
7390 \\
7400 \\
7410 \\
7420 \\
7430 \\
7440 \\
7450 \\
7455 \\
7470 \\
7475 \\
7480 \\
7490 \\
7495 \\
7500 \\
7509 \\
7510 \\
7520 \\
7526 \\
7528 \\
7540 \\
7550 \\
7558 \\
\end{array}$ & $\begin{array}{c}- \\
- \\
- \\
- \\
- \\
- \\
- \\
- \\
- \\
- \\
- \\
- \\
\text { a0.4 } \\
.90 \\
\text { a. } \\
\text { a.6 } \\
.21 \\
- \\
- \\
- \\
- \\
- \\
- \\
- \\
- \\
- \\
- \\
- \\
- \\
- \\
- \\
-\end{array}$ & 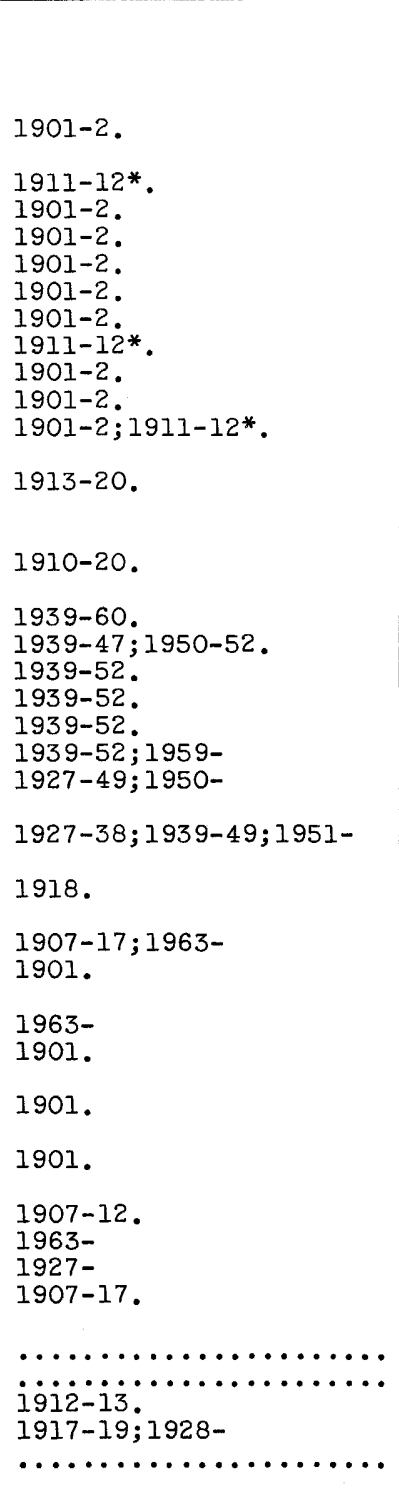 & $\begin{array}{l}1962- \\
1962-\end{array}$ & \\
\hline
\end{tabular}




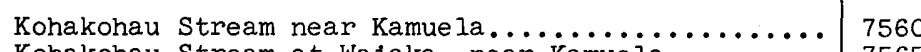
Kohakohau Stream at Walaka, near Kamuela............. 7565

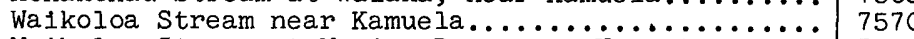

Waikoloa Stream at Marıne Dam, near Kamuela....... 7580

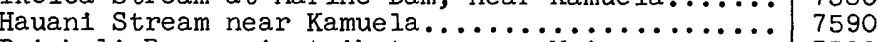

Paiakul1 Reservoir tributary near Waimea........ 7590.4

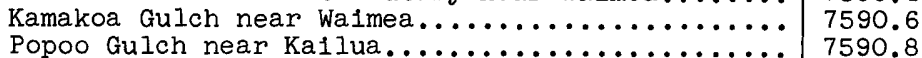

Wa1aha Stream:

Right Branch Walaha Stream near Holualoa........ 7592 Wa1aha Stream at Luawa1, near Holualoa.......... 7593

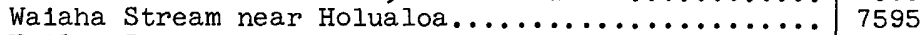

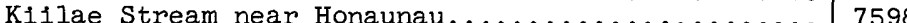
Waiohinu Springs, mauka station, near Naalehu... 7600 Wa1ohinu Springs maka Kahtl Kanlidpali

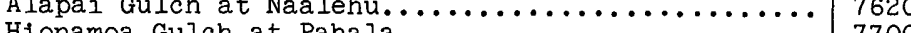

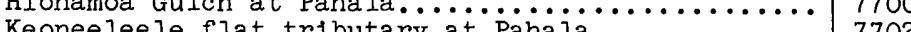
Keoneeleele flat tributary at Pahala............ 7702 Mariana Islands

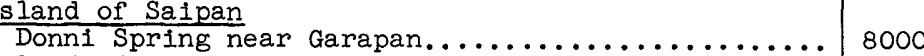

Island of Guam

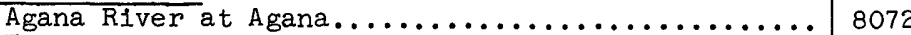

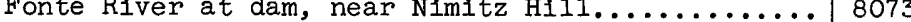

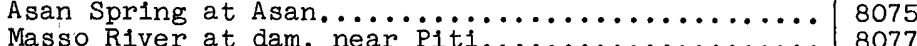

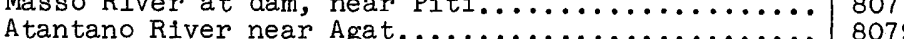

Santa Rita Spring at Santa Rita.............. 8080

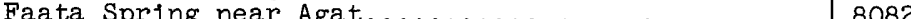

Finile River at Agat.

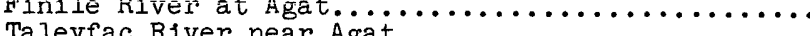

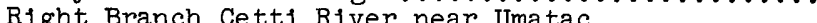

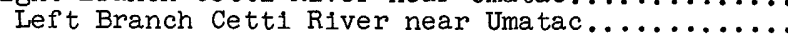

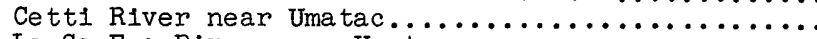

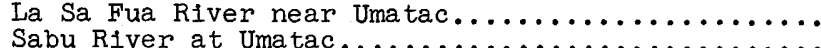

Right Branch Uma tac River at Uma tac $\ldots \ldots \ldots \ldots \ldots \ldots$

Left Fork of Left Branch Umatac River near $\cdots \cdots$

Right Fork of Left Branch Umatac River:

Piga Spring near Umatac.................... 8130

Right Fork of Left Branch Umatac River ä Umatac 8140

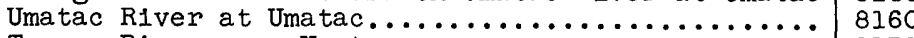

Toguan River near Umatac.................................... 8170

Geus River above Siligin Spring tributary, near 8200 Merizo.

Siligin Spring near Merizo................. 8205 Geus River below Siligin Spring tributary, near Merizo.

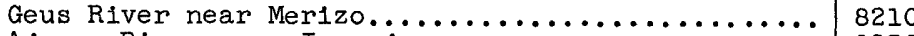

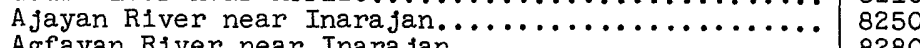

Right Fork of Right Branch Inarajan River near $\quad 8300$ Inarajan.

* Gage helghts, or gage helghts and discharge measurements only.

a Approximately.

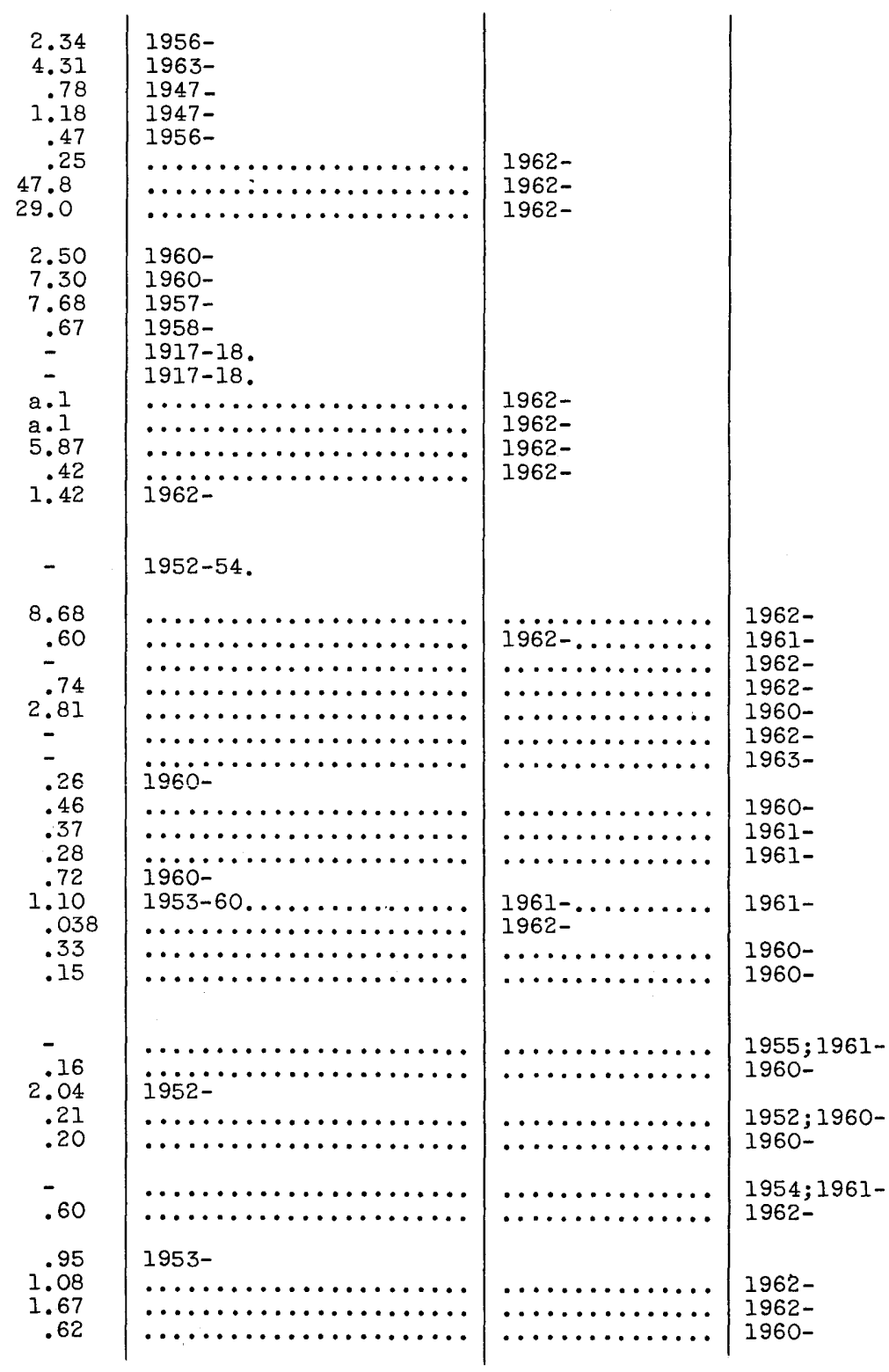




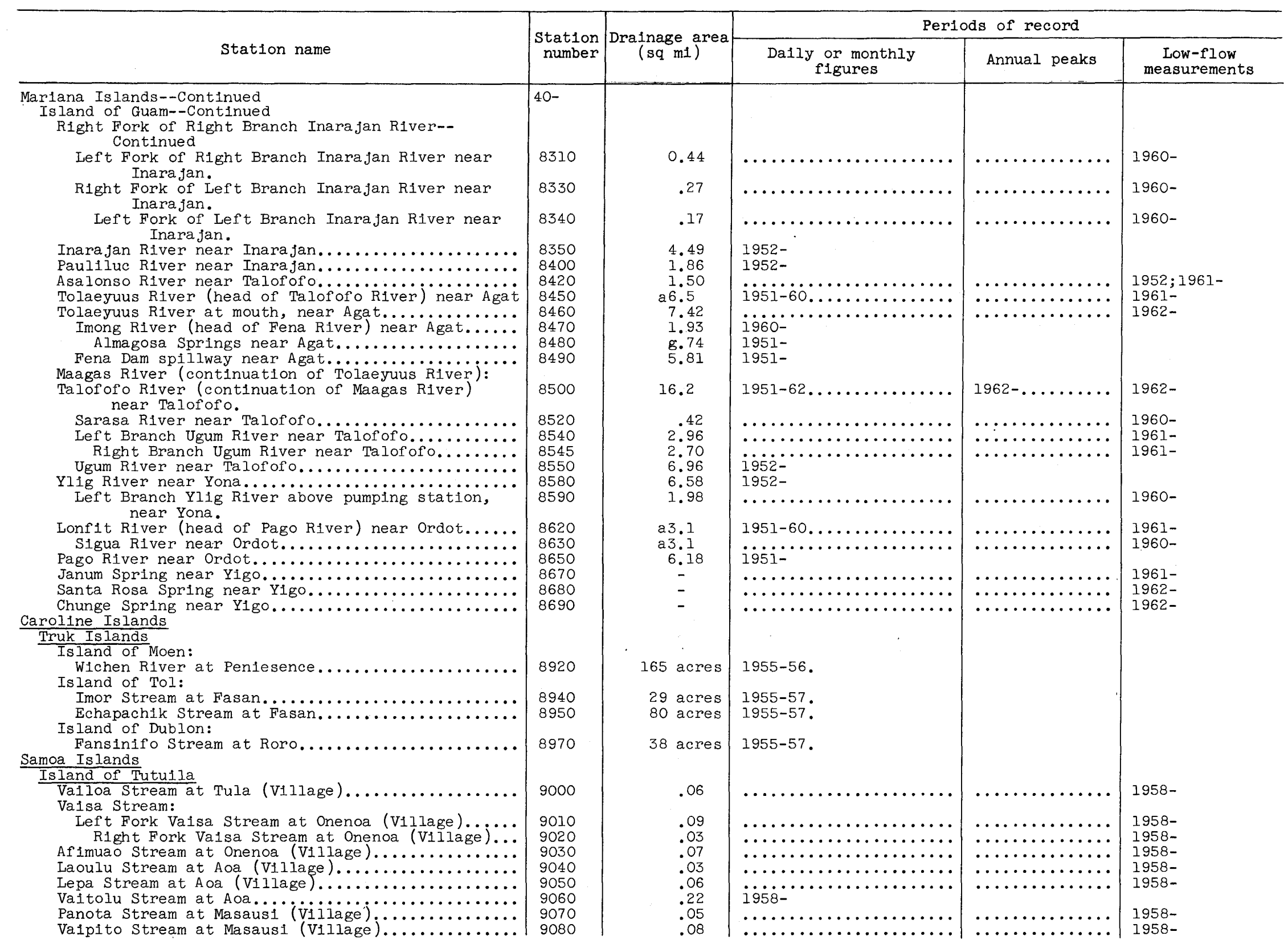


Tagau Stream at Masefau (Village)

Talaloa Stream at Masefau (Village)

Pago Stream tributary (Pago Stream) at Afono Village).

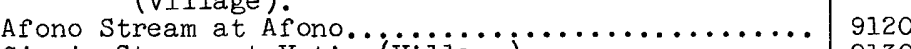

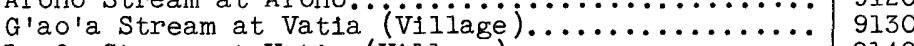

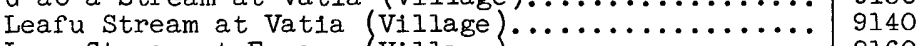

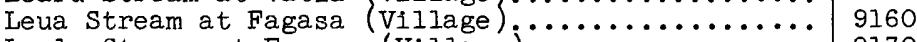

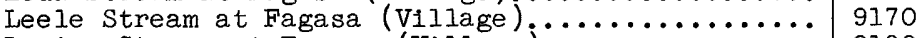

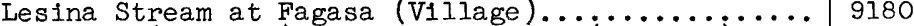

Leaveave (Fagafue) Stream near Aasu (Viliage)..... 9190

Upper Right Branch Leaveave Stream near Aasu.... 9192

Lower Right Branch Leaveave Stream near Aasu.... 9194

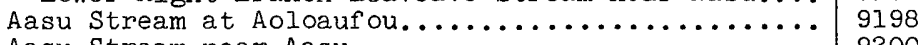

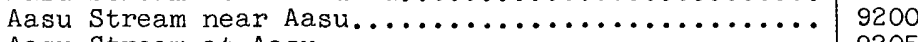

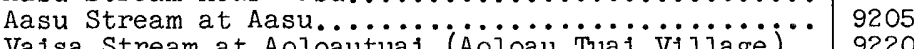

Valsa Stream at Aoloautual (Aoloau Tual Viliage) $j 9220$

Vallolo stream at Aoloautual (Aoloau Tua1 Village)

Maloata (Moloata) Stream at Maloata (Moloata.

$$
\text { Village). }
$$

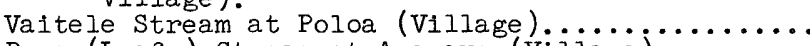

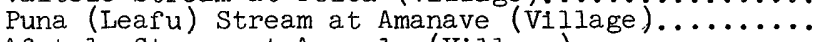

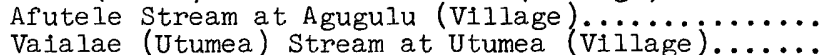

Utanuatele (Utuanua Iti1ti) Stream at Seetage (Village).

Soonapule (Utuanua Tele) Stream at Seetage (Village).

Atauloma Stream at Afao.

Asili Stream near Asili (Vijiage $\ldots \ldots \ldots \ldots \ldots \ldots$

Asili Stream at Asili (village

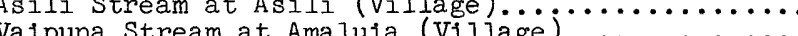

Leafu Stream near Leone (Village).

Vafue

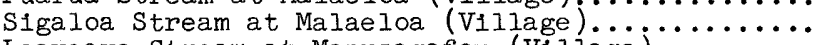

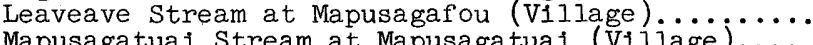

Mapusagatuai Stream at Mapusagatuai (Village) da1ry) near Mapusagatua1

Right Branch Taumata Stream (at old Government dairy) near Mapusagatuai.

Right Branch Vaitele Stream at Tafuna (Village)..

Left Branch Vaitele Stream at Tafuna (Village)..

Sauino (Asofitu) Stream near Nuuuli (Viliage)....

Matali (Papa) Stream near Nuuuli (village $\{\ldots \ldots$

Leele (Tauese) Stream near Nuuuli (Village).......

Avau Stream at Faganeanea (Village).

Afu Stream at Faganeanea (Village) $\ldots \ldots \ldots \ldots \ldots$

(Matuu) Stream at Matuu.

$\ldots \ldots \ldots \ldots$ Stream) at Fagaalu (Village).

Faga'alu Stream near Fagaalu..................................... 949

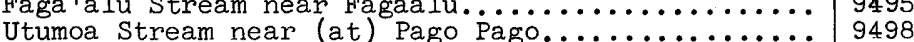

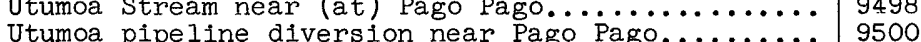

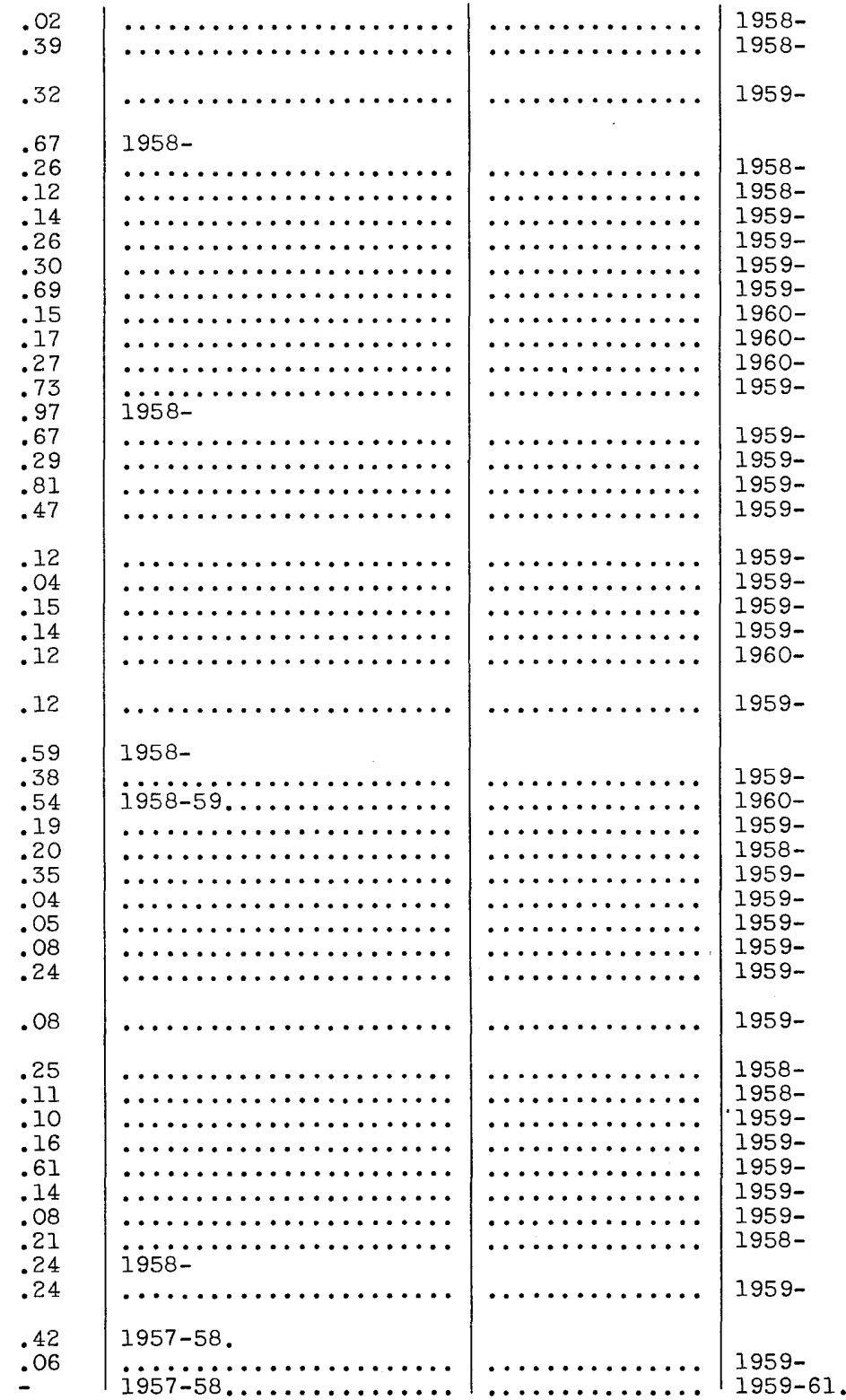

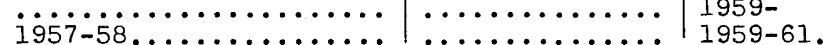

g Hydrologic drainage boundary uncertain owing to ground-water exchange. 


\begin{tabular}{|c|c|c|c|c|c|}
\hline \multirow[b]{2}{*}{ Station name } & \multirow{2}{*}{$\begin{array}{r}\text { Station } \\
\text { number }\end{array}$} & \multirow{2}{*}{$\begin{array}{c}\text { Drainage area } \\
(\mathrm{sq} \mathrm{m} 1)\end{array}$} & \multicolumn{3}{|c|}{ Periods of record } \\
\hline & & & $\begin{array}{c}\text { Daily or monthly } \\
\text { figures }\end{array}$ & Annual peaks & $\begin{array}{l}\text { Low-flow } \\
\text { measurements }\end{array}$ \\
\hline 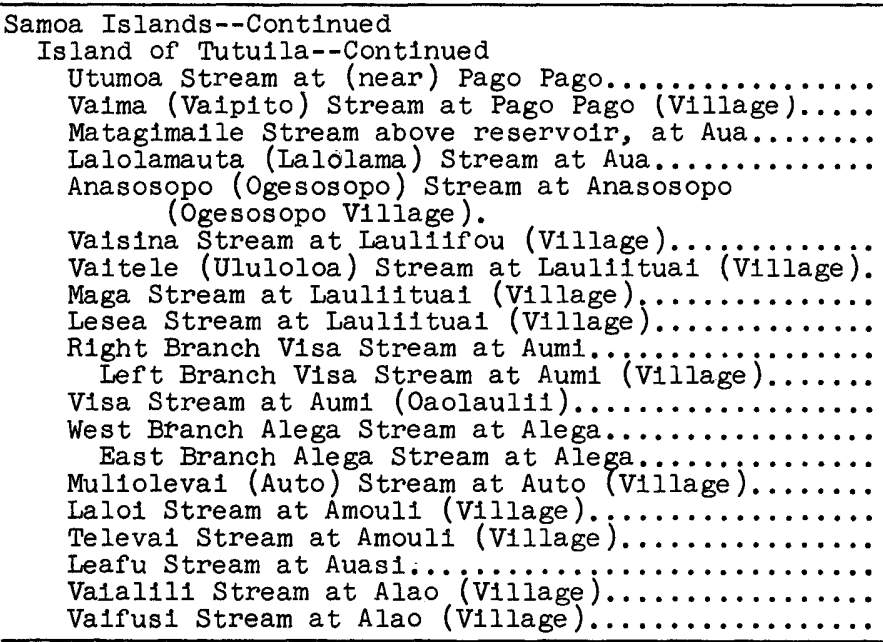 & $\begin{array}{l}40- \\
9505 \\
9510 \\
9530 \\
9535 \\
9550 \\
9555 \\
9560 \\
9565 \\
9570 \\
9580 \\
9585 \\
9590 \\
9600 \\
9605 \\
9610 \\
9620 \\
9630 \\
9640 \\
9650 \\
9660\end{array}$ & $\begin{array}{l}0.13 \\
.09 \\
.09 \\
.10 \\
.05 \\
.04 \\
.05 \\
.21 \\
.17 \\
.05 \\
.02 \\
.14 \\
.16 \\
.16 \\
.14 \\
.08 \\
.10 \\
.55 \\
.05 \\
.02\end{array}$ & 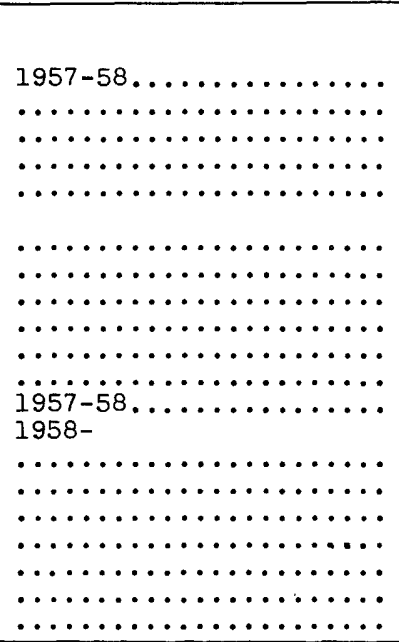 & 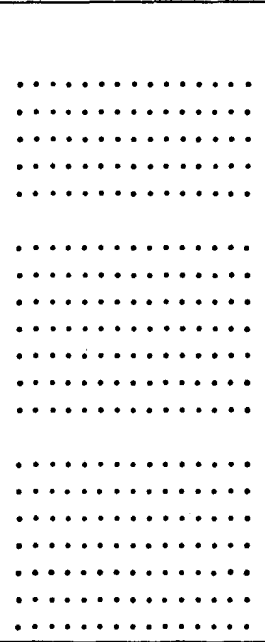 & $\begin{array}{l}1959- \\
1959- \\
1959- \\
1959- \\
1959- \\
1959- \\
1959- \\
1959- \\
1959- \\
1958- \\
1958- \\
1963- \\
1958- \\
1958- \\
1958- \\
1958- \\
1958- \\
1958- \\
1958-\end{array}$ \\
\hline
\end{tabular}


Page

Aasu Stream.

Afimuao Stream.

Afono Stream.

Afu Stream...

Afuelo Stream.

Afutele Stream.

Agana River...

Agfayan River.

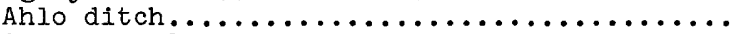

Ahualoa Gulch.

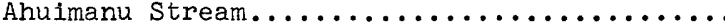

Ajayan River.

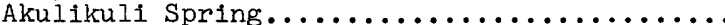

Alákahi Stream.

Alapai Gulch.

Alega Stream, East Branch.

Alega Stream, West Branch.

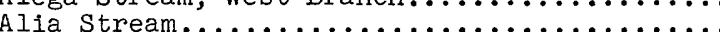

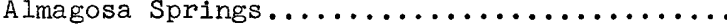

Alo Stream.

Amaile Stream.

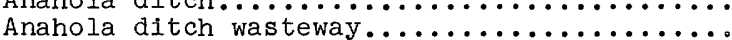

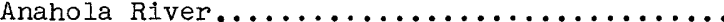

Anahola Stream.

Anahulu River.

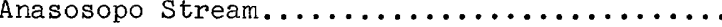

Asalonso River.

Asan Spring.

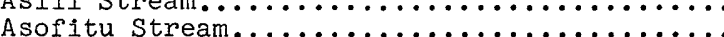

Atantano River.

Atauloma Stream.

Auto Stream.

Avau Stream.

Awini ditch.

Center ditch

Cetti River....

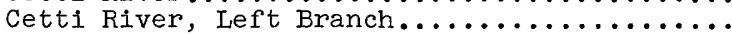

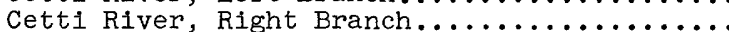

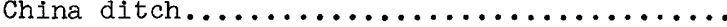

Chunge Spring.

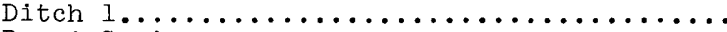

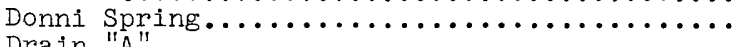

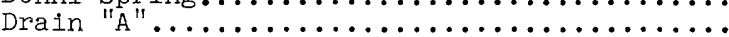

East Branch or Fork. See name of main stream.

East Honokane Iki intake.

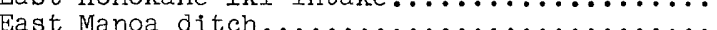

East Wailuaiki Stream.

East Wailuanui Stream.

Echapachik Stream...

Faata Spring...

Faga'alu Stream.

Fagafue Stream.

Fansinifo Stream.

Fena Dam spillway.

Finile River.

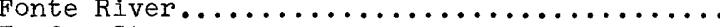

Fuafua Stream.

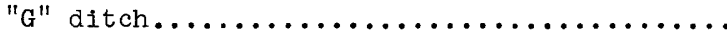

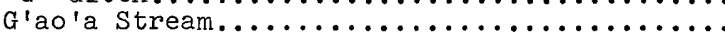

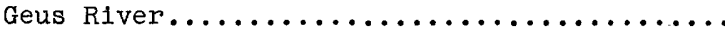

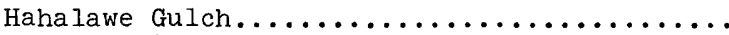
Hahalawe Stream, Right Branch............ Haiku ditch.

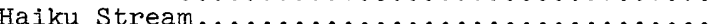

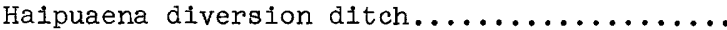

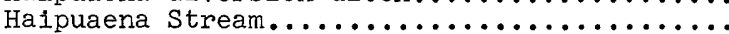

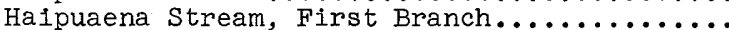

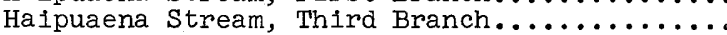

Halaulani Stream.

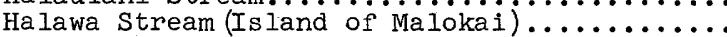

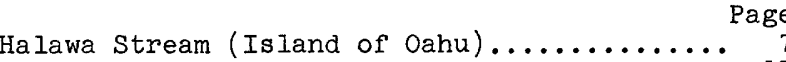

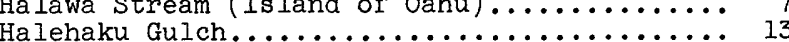

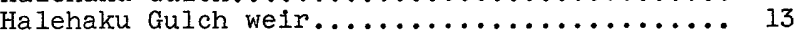

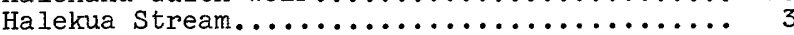

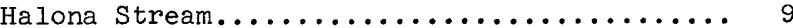

Hamakua ditch................... 16

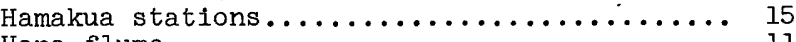

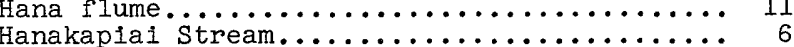

Hanakoa Stream. $\ldots \ldots \ldots \ldots \ldots \ldots \ldots \ldots \ldots \ldots \ldots \ldots \ldots \ldots \ldots \ldots \ldots_{6}$

Hanalei ditch........................ 5

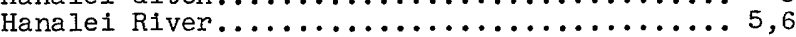

Hanalei tunnel outlet................. 5

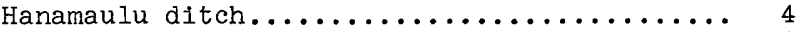

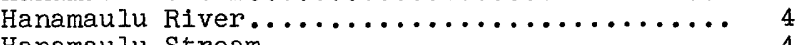

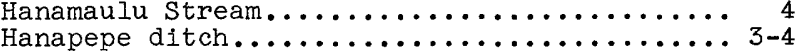

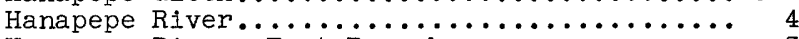

Hanapepe River, East Branch............ 3

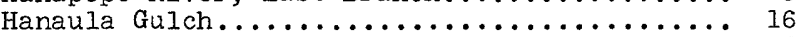

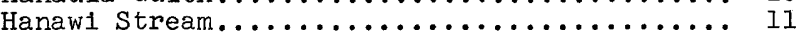

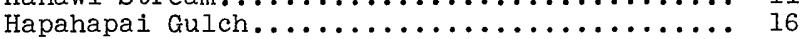

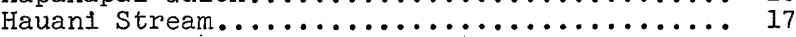

Hawaiian Electric Co tunnel............... 7

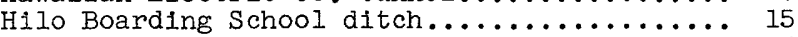

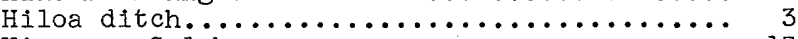

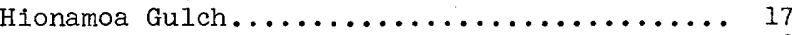

Hoea Stream........................ 6

Honokane Nui Stream, East Branch............. 16

Honokane Nui Stream, West Branch............... 16

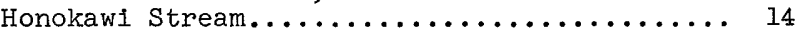

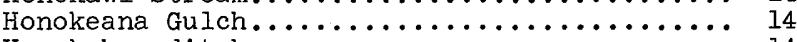

Honokohau ditch.................... 14

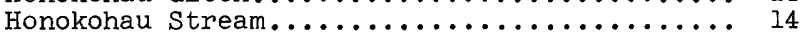

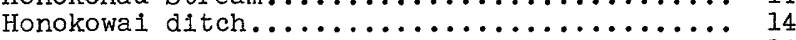

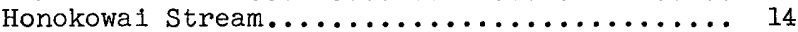

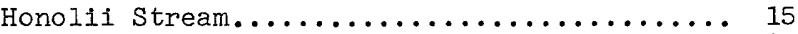

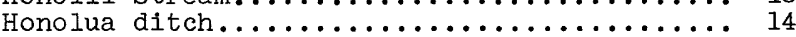

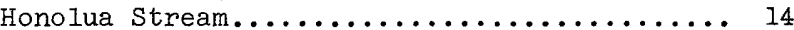

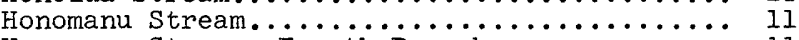

Honomanu Stream, Fourth Branch................ 11

Honomanu Stream, Seventh Branch............. 11

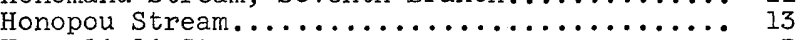

Honouliuli stream........................ 7

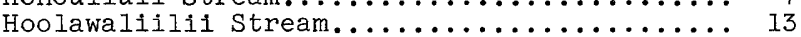

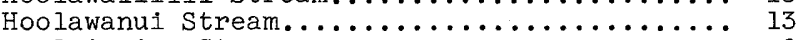

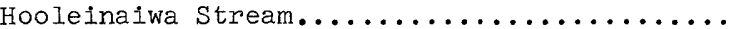

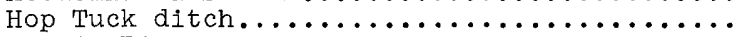

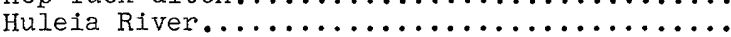

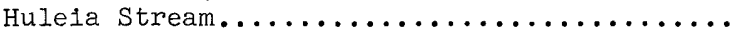

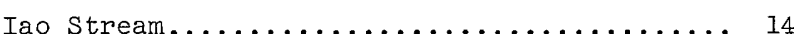

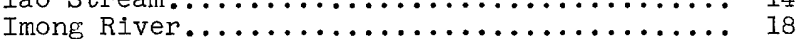

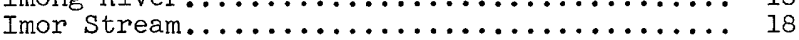

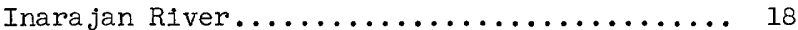

Inarajan River, Left Fork of Left Branch..... 18

Inara jan River, Left Fork of Right Branch.... 18

Inara jan River, Right Fork of Left Branch.... 18

Inara jan River, Right Fork of Right Branch... 17

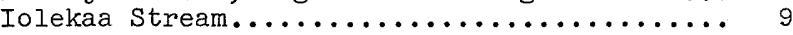

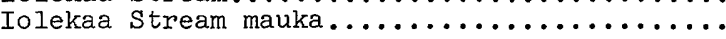

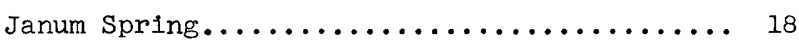

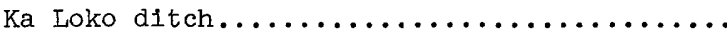

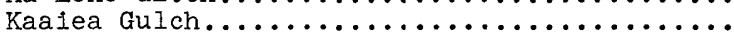

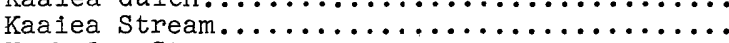

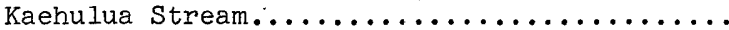

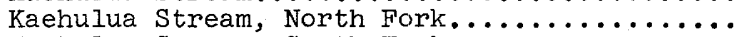

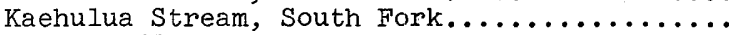

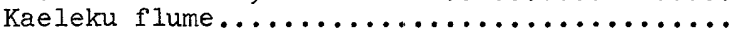

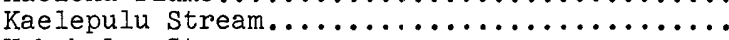

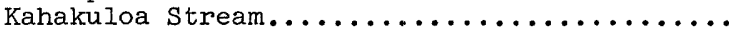

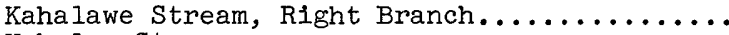

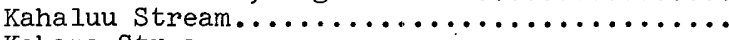

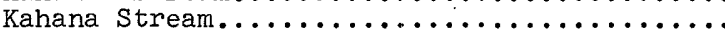

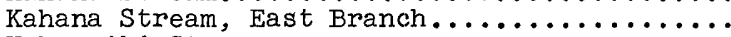

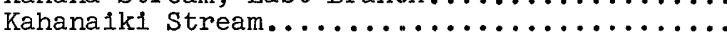

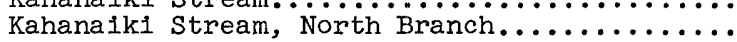

.




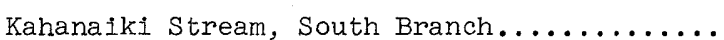

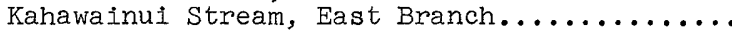

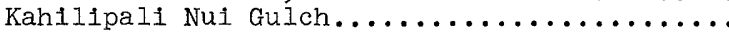

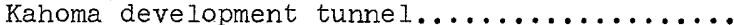

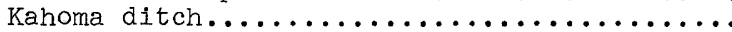

Kahoma Stream.

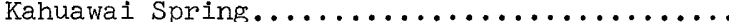

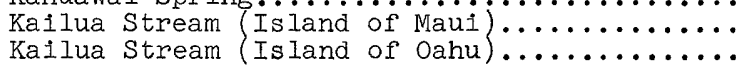

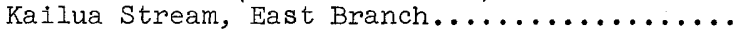

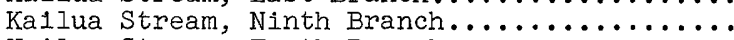

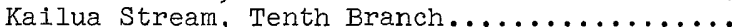

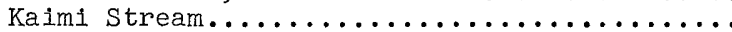

Kaimu stream.

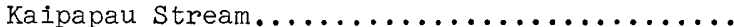

Kakaako Gulch.

Kala lau Stream.

Kalaoa Stream.

Kalauao Stream.

Kalihi Stream.

Kalihiwai ditch

Kalihiwai River.

Kaluanui ditch.

Kaluanui Stream.

Kaluapeelua Gulch.

Kaluapulani Gulch

Kamaka lepo Stream.

Kamakoa Gulch....

Kamananui Stream.

Kamooloa Stream.

Kanaha ditch.

Kaneha ditch.

Kaneohe Stream.

Kapaa ditch

Kapaa River.

Kapaa Stream.

Kapahi ditch...

Kapaloa Stream.

Kapaula Gulch.

Kapaula Stream.

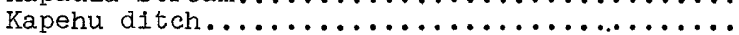

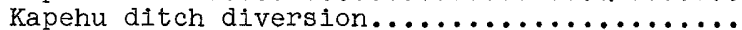

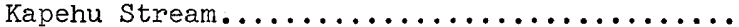

Kapuna Stream.

Kauaikinana Stream.....

Kauaula ditch.

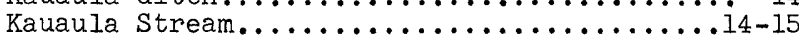

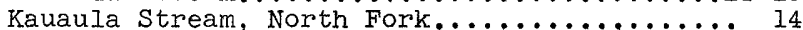

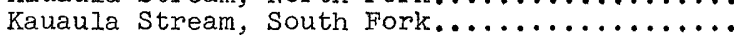

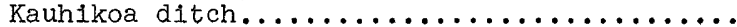

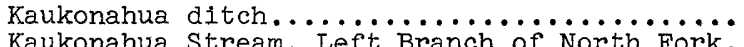

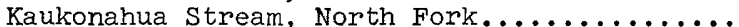

Kaukonahua Stream, Right Branch of North

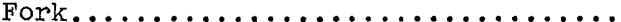

Kaukonahua Stream, Right Branch of South Fork.

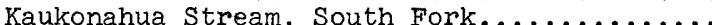

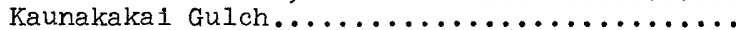

Kaunakakai Stream.

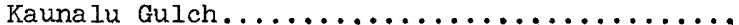

Kaupuni Stream.

Kawa Stream.

Kawaikoi Stream.

Kawainui Ganal.

Kawainui River.

Kawainui Stream (İ iand of Hawaij)..........

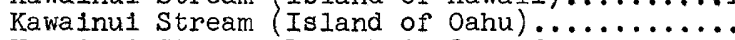

Kawa inu1 Stream, Branch 1, 2, and $3 \ldots \ldots \ldots$ 15-16

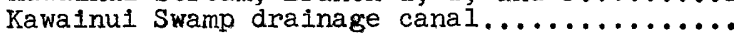

Kawela Gulch, East Fork.

Kawela Gulch, Right Branch of East Fork......

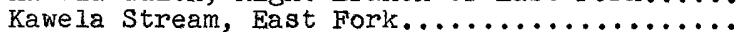

Kawela Stream, Right Branch of East Fork.....

Kawi Stream.

Keaahala Stream.

Keahua Stream.

Keehia Gulch.
Page

10

14

14

7
13
-8

8

13

13

8
16
13

10
10

10
6

15

7
7
5

5

13

10

14

8

10

8,9

$\begin{array}{r}4 \\ 4 \\ \hline\end{array}$

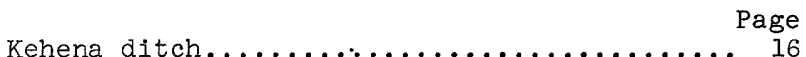

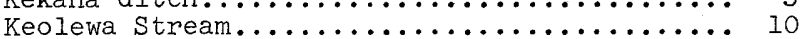

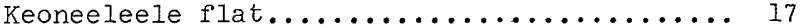

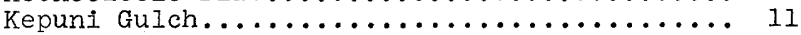

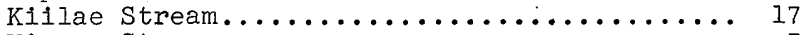

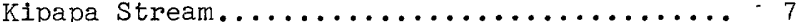

Koaie Stream................. 3

Kohakohau Stream..................... 17

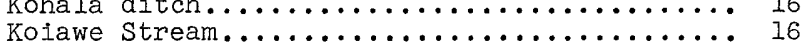

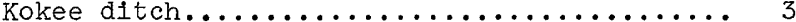

Koloa ditch ..................... 4

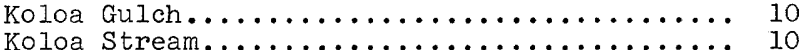

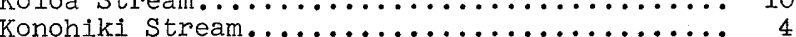

Koolau ditch (Island of Mauij ............... 12

Koolau ditch (Island of Oahu ............... 6

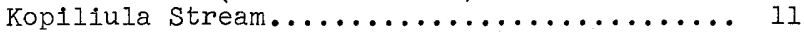

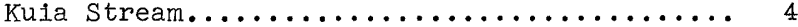

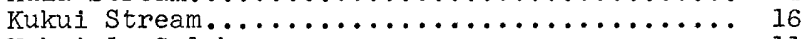

Kukuiula Gulch.................... II

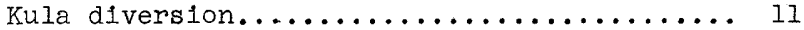

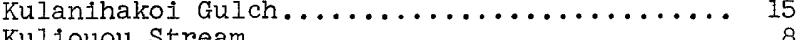

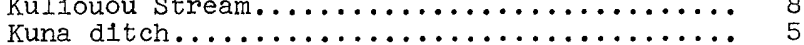

Kuou ditch $\ldots \ldots \ldots \ldots \ldots \ldots \ldots \ldots \ldots \ldots \ldots \ldots$. 8

Kuou stream..................... 8

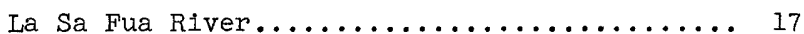

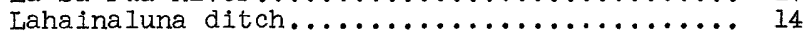

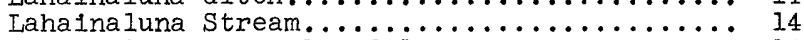

Lahainaluna weirs 1 and $2 \ldots \ldots \ldots \ldots \ldots \ldots 14$

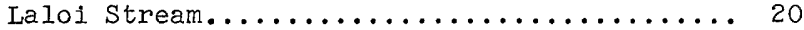

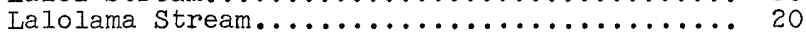

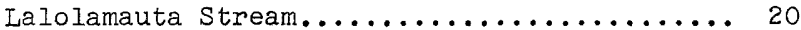

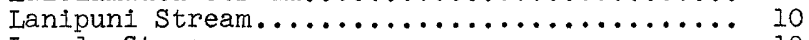

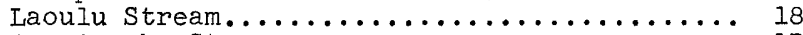

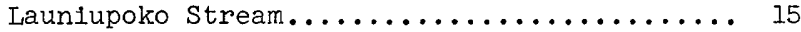

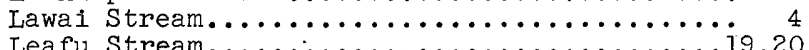

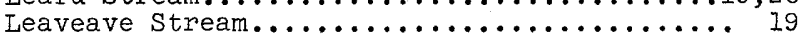

Leaveave Stream, Lower Right Branch............ 19

Leaveave Stream, Upper Right Branch........ 19

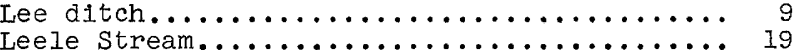

Left Branch or Fork. See name of main stream.

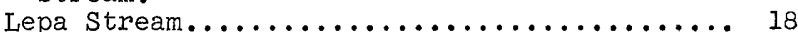

Lesea Stream. $_{1} \ldots \ldots \ldots \ldots \ldots \ldots \ldots \ldots \ldots \ldots \ldots \ldots \ldots \ldots{ }_{20}$

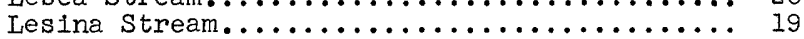

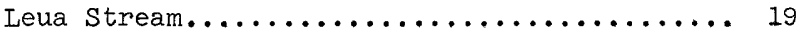

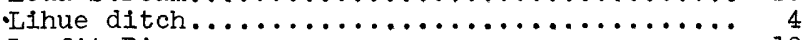

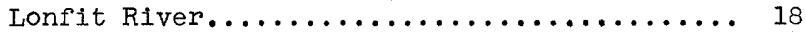

Lower Anahola ditch................. 5

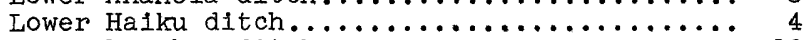

Lower Hamakua ditch.................. 16

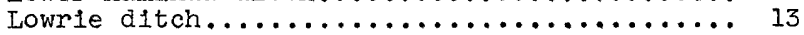

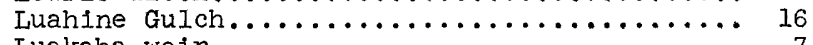

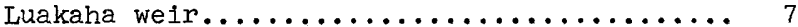

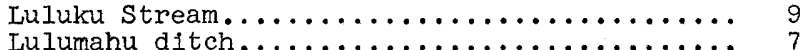

Lumahai River...................... 6

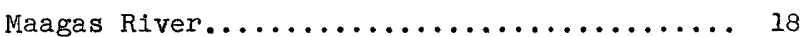

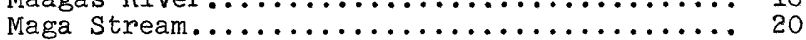

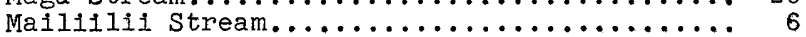

Main Spring..................... 8

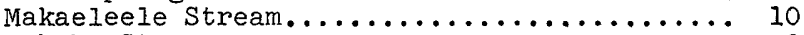

Makaha stream.......................... 6

Maka leha ditch..................... 5

Maka leha stream......................... 6

Makamakaole Stream, Left Branch........... 14

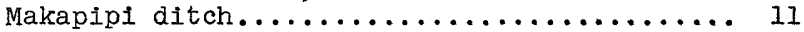

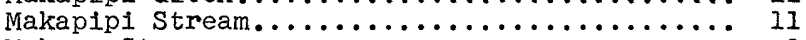

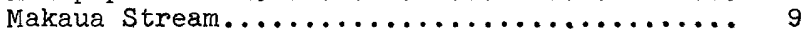

Makawao ditch.................... 8

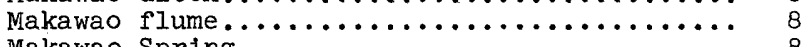

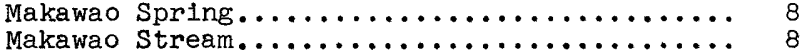


Page

Makaweli River

Makua Stream.

Ma laekahana Siream.

Malaekahana Stream,

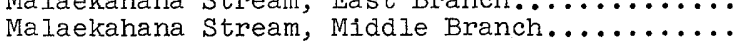

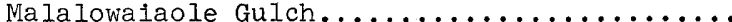

Maloata Stream.

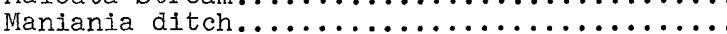

Manoa Stream.

Manoa Stream, East Branch.

Manoa Stream, West Branch.

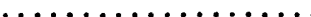

Manuel Luis ditch.

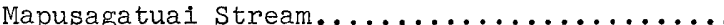

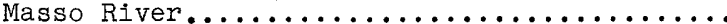

Matali Stream.

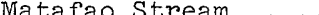

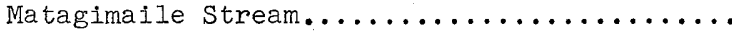

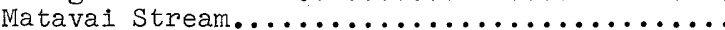

Matuu Stream.

Mauka ditch

Maunawili ditch.

Middle Branch or Fork. Se name of main stream.

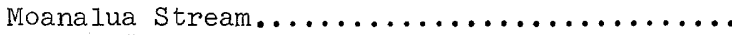

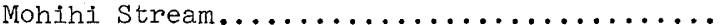

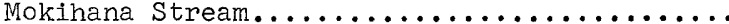

Mokomoko Gulch.

Moloata Stream

Moole ditch.

Moomoonui Gulch.

Nahomalu Stream.

Naililithaele Stream.

Nailijilhaele Stream Second Branch $\ldots . . .$.

New Hamakua ditch (Island of Hawai1).............

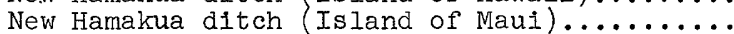

North Branch or Fork. See name of main stream.

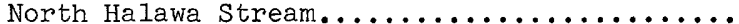

North Luluku ditch.

North Waiehu ditch.

North Waiehu Stream.

North Wailua ditch.

Nuuanu Stream.

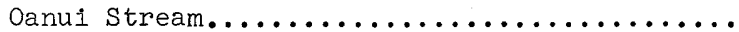

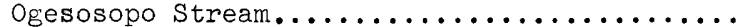

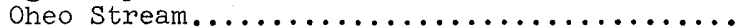

Oio Stream.

Olaa flume ...........

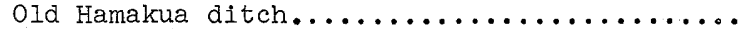

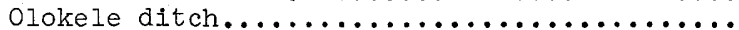

Olokele River.

Olowalu ditch.

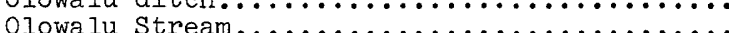

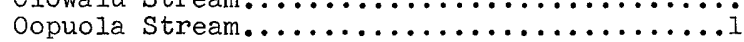

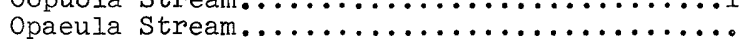

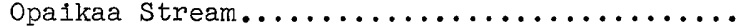

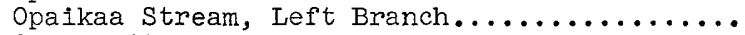

Opana ditch.

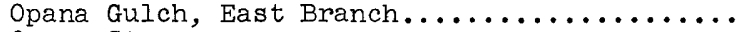

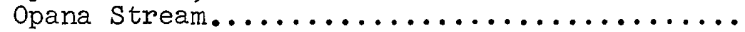

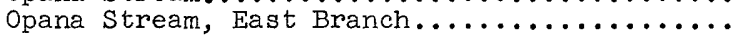

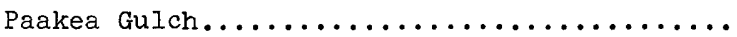

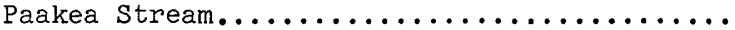

Paauhu Gulch.

Pago River..

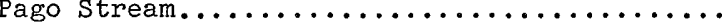

Paiakuli Reservoir

Palikea Stream.

$\mathrm{Palolo}$ ditch

Palolo Stream.

Panota Stream.

Paopao Stream.

Papa Stream.

\section{6
10
10
10
15
19
14
8
7
7
4
12
19
17
19
19
20
19
19
6
8
8

7
3
3
10
19
7
11
7
20}

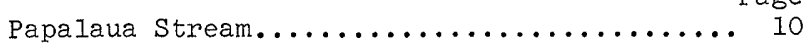

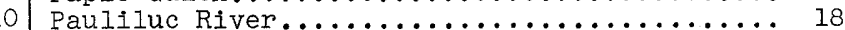

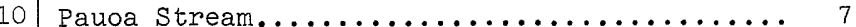

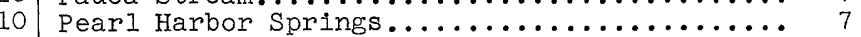

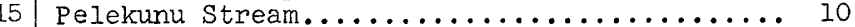

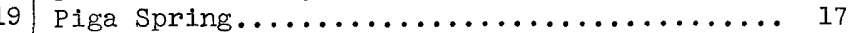

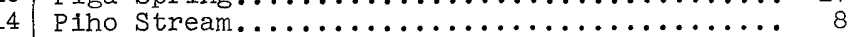

8 Piihonua Stations Nos. $1-4 \ldots \ldots \ldots \ldots \ldots \ldots . \ldots 15$

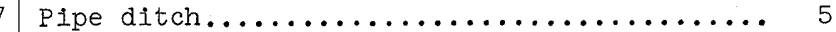

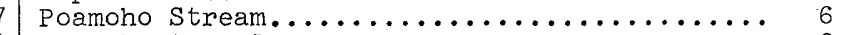

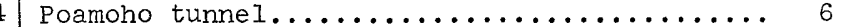

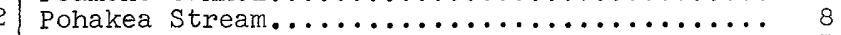

9 Pohakuhonu stream................... 5

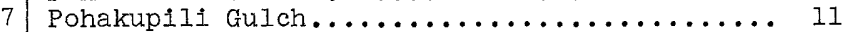

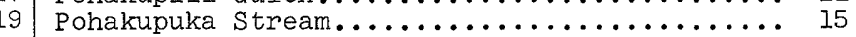

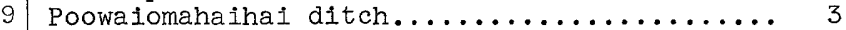

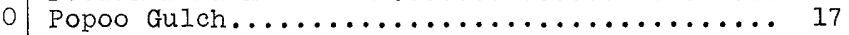

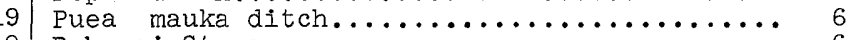

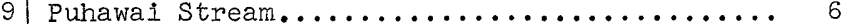

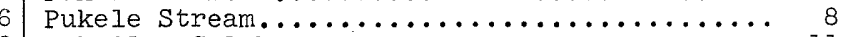

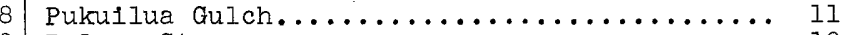

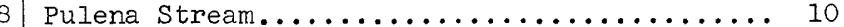

Pump ditch.................................

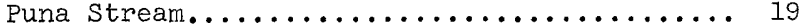

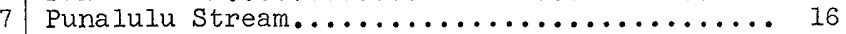

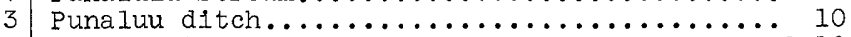

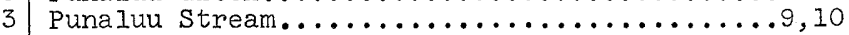

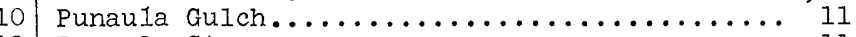

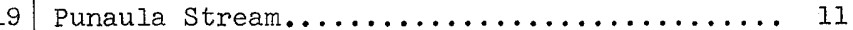

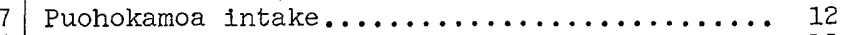

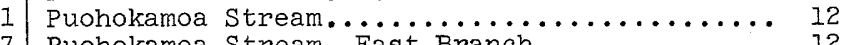

7 Puohokamoa Stream, East Branch............ 12

20 Puohokamoa Stream, Middle Branch......... 12

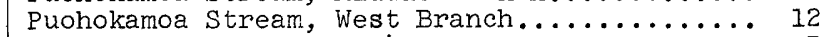

6
13

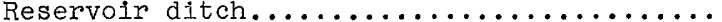

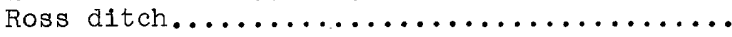

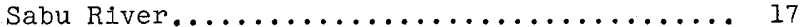

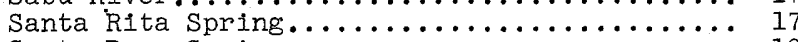

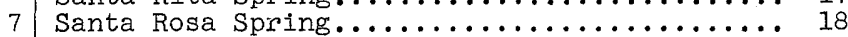

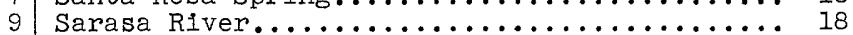

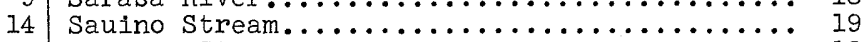

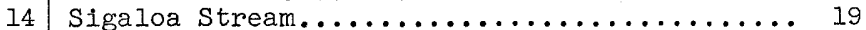

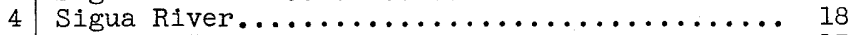

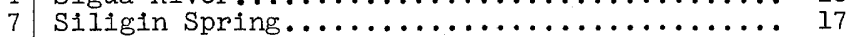

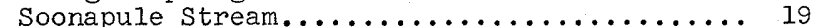

13 South Branch or Fork. See name of main

20 stream.

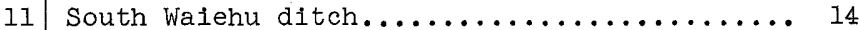

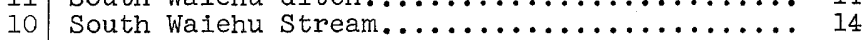

15 Spreckels ditch........................ $12, i 3,14$

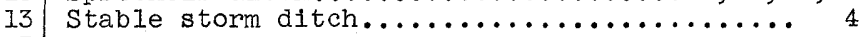

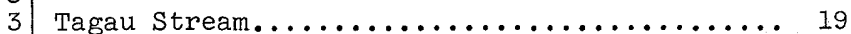

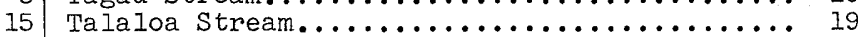

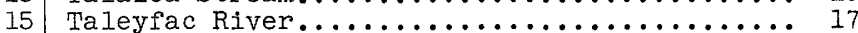

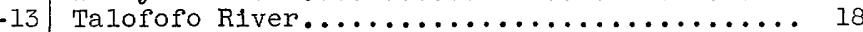

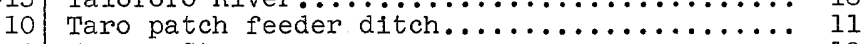

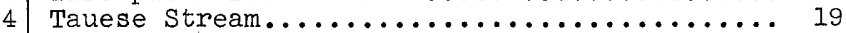

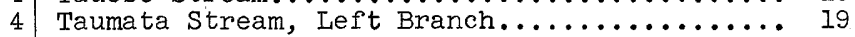

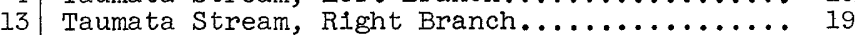

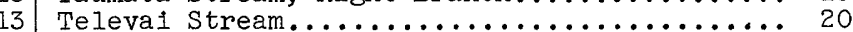

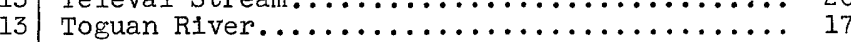

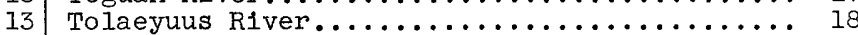

11

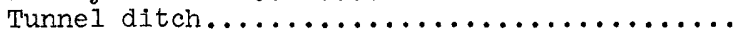

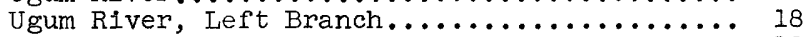

Ugum River, Right Branch............... 18

Uhau oile stream.................... 4

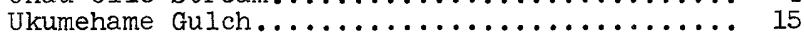

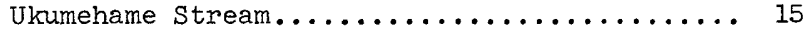

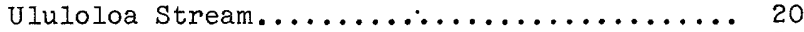

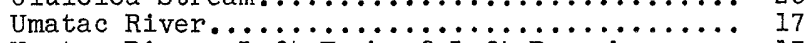

Umatac River, Left Fork of Left Branch...... 17

Umatac River, Right Branch................ 17

Umatac River, Right Fork of Left Branch...... 17 


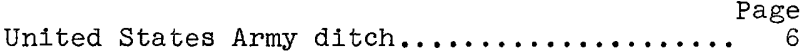

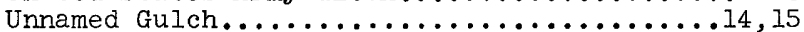

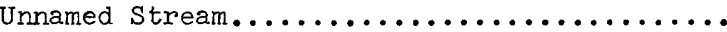

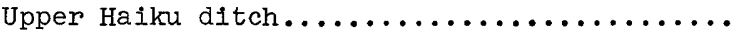

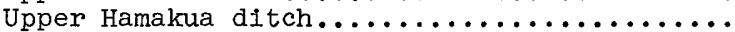

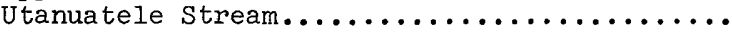

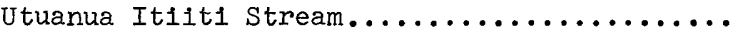

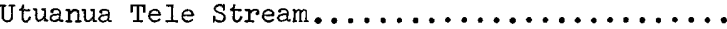

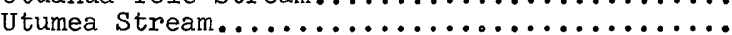

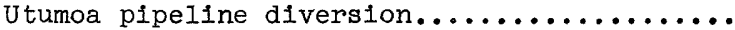

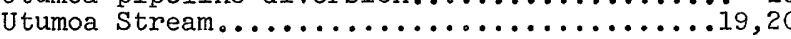

Valalae Stream.

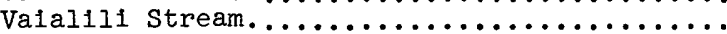

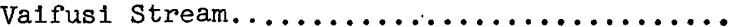

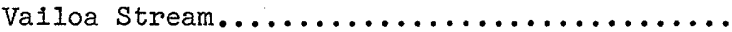

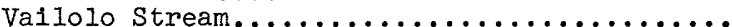

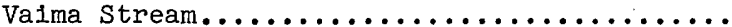

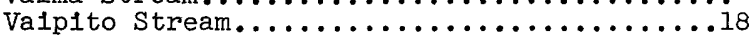

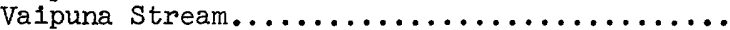

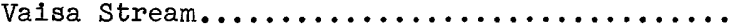

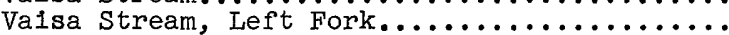

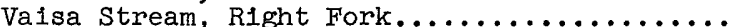

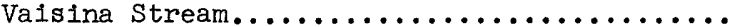

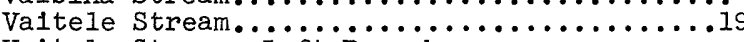

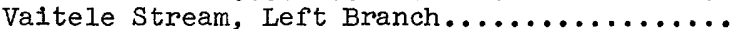

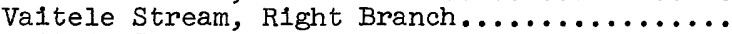

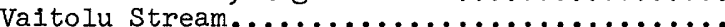
Visa Stream.......................

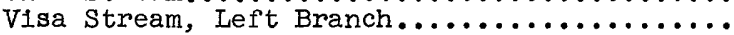
Visa Stream, Right Branch...............

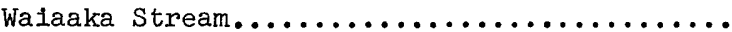

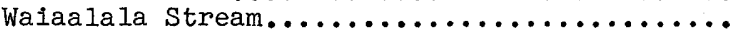

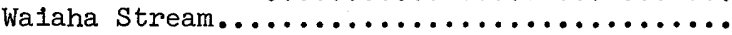

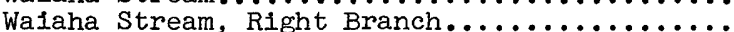

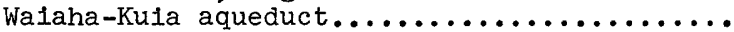

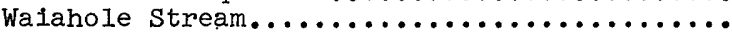

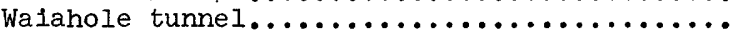

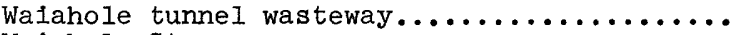

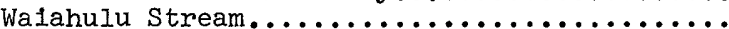

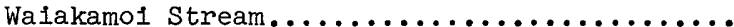

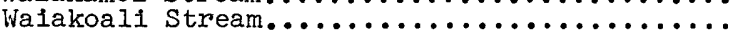

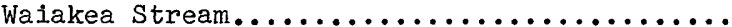

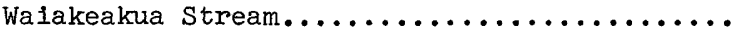

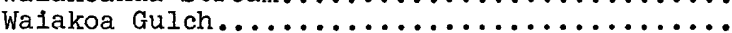

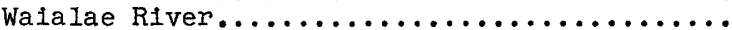

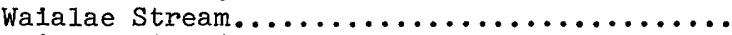

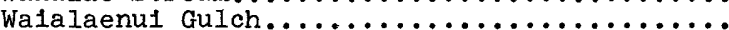

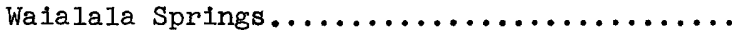

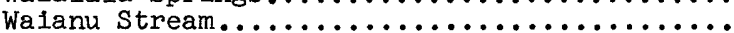

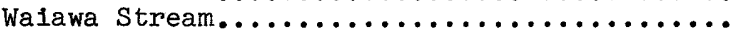

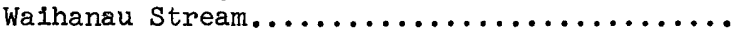

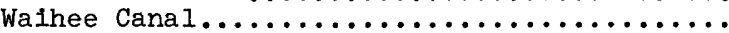

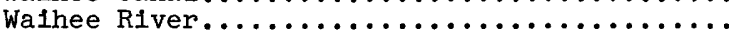

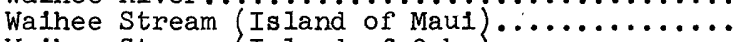

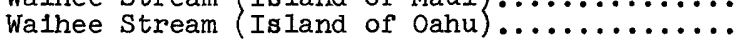

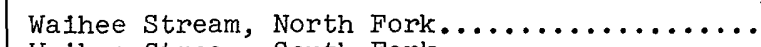

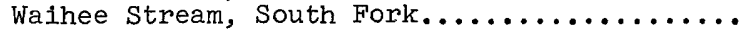

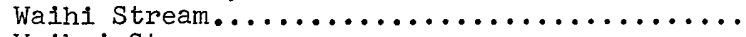

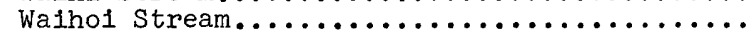

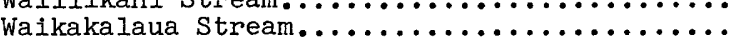

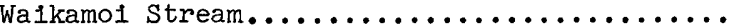

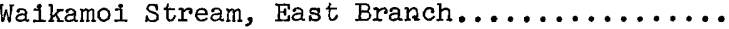

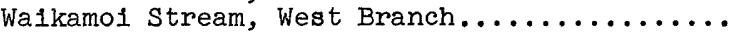

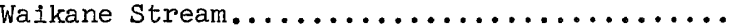

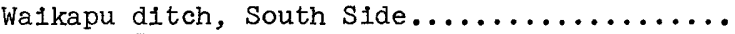

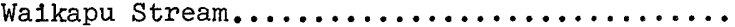

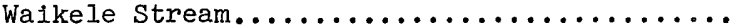

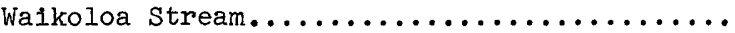

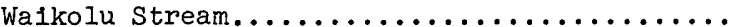

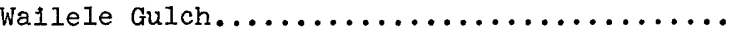

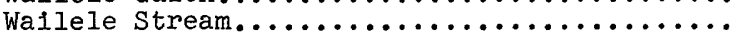

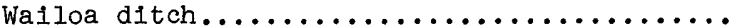
Wailua ditch $\ldots \ldots \ldots \ldots \ldots \ldots \ldots \ldots \ldots \ldots \ldots$

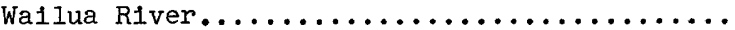
Wallua River, East Branch of North Fork......

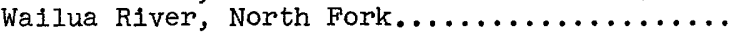

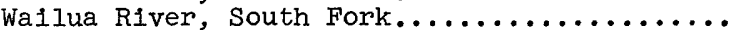

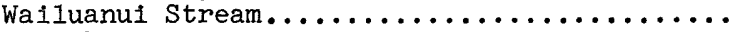

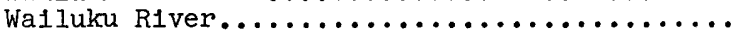

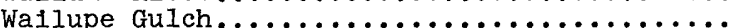

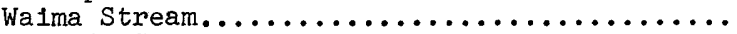

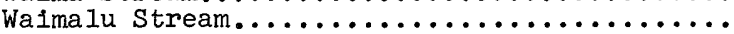

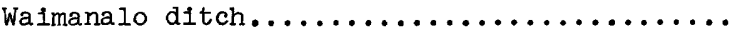

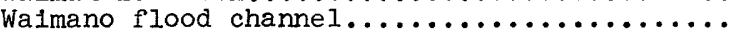

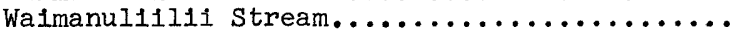

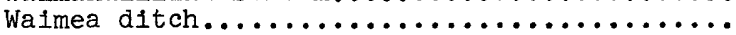

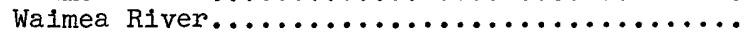

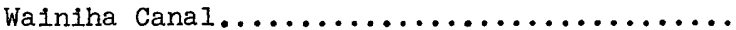

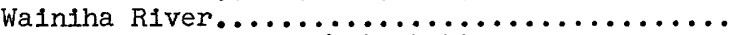

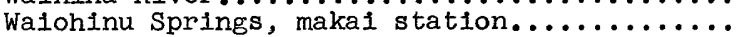

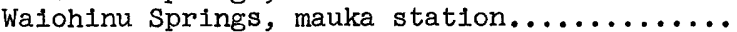

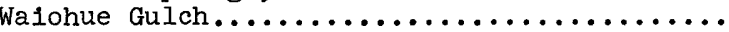

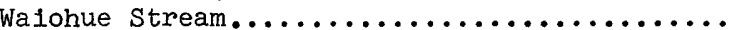

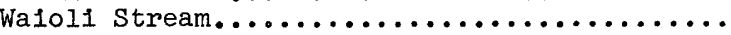

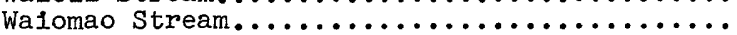

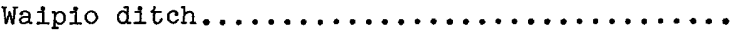

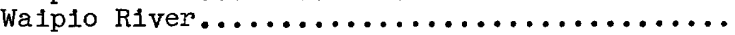

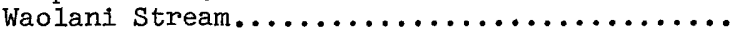

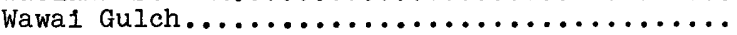
West Branch or Fork. See name of main stream.

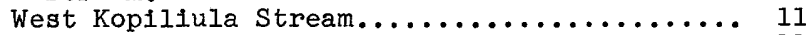

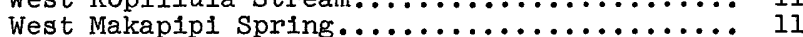

West Wailuaiki Stream.................... II

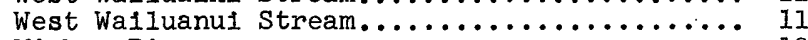

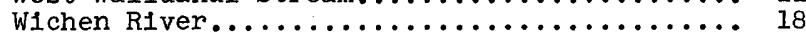

Wing wo Ta1 ditch.................. 9

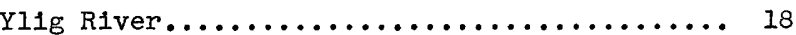

Ylig River, Left Branch....................... 18

Young Mau ditch $\ldots \ldots \ldots \ldots \ldots \ldots \ldots \ldots \ldots \ldots \ldots \ldots$ 

